\title{
Safety, tolerability and pharmacodynamics of apical sodium-dependent bile acid transporter inhibition with volixibat in healthy adults and patients with type 2 diabetes mellitus: a randomised placebo- controlled trial
}

Renger G. Tiessen ${ }^{1 *}$ D , Ciara A. Kennedy², Bradley T. Keller², Nancy Levin², Lisette Acevedo², Bronislava Gedulin², Andre A. van Vliet ${ }^{1}$, Alejandro Dorenbaum ${ }^{2}$ and Melissa Palmer ${ }^{3}$

\begin{abstract}
Background: Pathogenesis in non-alcoholic steatohepatitis (NASH) involves abnormal cholesterol metabolism and hepatic accumulation of toxic free cholesterol. Apical sodium-dependent bile acid transporter (ASBT) inhibition in the terminal ileum may facilitate removal of free cholesterol from the liver by reducing recirculation of bile acids (BAs) to the liver, thereby stimulating new BA synthesis from cholesterol. The aim of this phase 1 study in adult healthy volunteers (HVs) and patients with type 2 diabetes mellitus (T2DM) was to assess the safety, tolerability, pharmacokinetics and pharmacodynamics of ASBT inhibition with volixibat (SHP626; formerly LUM002).

Methods: Participants were randomised 3:1 to receive once-daily oral volixibat $(0.5 \mathrm{mg}, 1 \mathrm{mg}, 5 \mathrm{mg}$ or $10 \mathrm{mg}$ ) or placebo for 28 days in two cohorts (HV and T2DM). Assessments included safety, faecal BA and serum 7a-hydroxy-4-cholesten-3-one (C4; BA synthesis biomarker).
\end{abstract}

Results: Sixty-one individuals were randomised (HVs: placebo, $n=12$; volixibat, $n=38$; T2DM: placebo, $n=3$; volixibat, $n=8)$. No deaths or treatment-related serious adverse events were reported. Mild or moderate gastrointestinal adverse events were those most frequently reported with volixibat. With volixibat, mean total faecal BA excretion on day 28 was 1.6-3.2 times higher in HVs (643.73-1239.3 $\mu \mathrm{mol} / 24 \mathrm{~h})$ and $~ 8$ times higher in T2DM (1786.0 $\mu \mathrm{mol} / 24 \mathrm{~h})$ than with placebo (HVs: $386.93 \mu \mathrm{mol} / 24$ h; T2DM: $220.00 \mu \mathrm{mol} / 24 \mathrm{~h}$ ). With volixibat, mean C4 concentrations increased by 1.3-5.3-fold from baseline to day 28 in HVs and by twofold in T2DM.

Conclusions: Volixibat was generally well tolerated. Increased faecal BA excretion and serum C4 levels support the mechanistic rationale for exploring ASBT inhibition in NASH. The study was registered with the Dutch clinical trial authority (Centrale Commissie Mensgebonden Onderzoek; trial registration number NL44732.056. 13; registered 24 May 2013).

Keywords: Apical sodium-dependent bile acid transporter, Bile acids, Clinical pharmacology, Non-alcoholic steatohepatitis, Non-alcoholic fatty liver disease, Phase 1 clinical trial, LUM002, SHP626, Type 2 diabetes mellitus, Volixibat

\footnotetext{
* Correspondence: tiessenrenger@prahs.com

${ }^{1}$ Early Development Services, Pharmaceutical Research Associates (PRA)

Health Sciences, Van Swietenlaan 6, 9728 NZ Groningen, PO Box 8144, 9702

Groningen, KC, Netherlands

Full list of author information is available at the end of the article
} 


\section{Background}

In 2014, the World Gastroenterology Organisation estimated that approximately 6 million individuals in the United States (US) had non-alcoholic steatohepatitis (NASH), of whom approximately 600,000 had NASHrelated cirrhosis [1]. However, the prevalence of NASH is difficult to establish because definitive diagnosis requires an invasive biopsy for histological confirmation. In a prospective ultrasound study of 400 asymptomatic adults in the US, NASH was confirmed by liver biopsy in $12 \%$ of individuals in the overall study population; this value increased to $22 \%$ in patients with diabetes mellitus [2]. The authors of this study also reported that $2.7 \%$ of patients had evidence of advanced fibrosis (stages 2-4) and, based on this proportion, estimated that more than 2 million middle-aged adults in the US may have undiagnosed advanced NASH.

Non-alcoholic fatty liver disease (NAFLD) ranges from simple steatosis, which is typically non-progressive, to $\mathrm{NASH}$ [3], which is characterised by steatosis in addition to inflammation and liver injury (hepatocyte ballooning with or without fibrosis) [4]. Among prospective studies with long-term histological follow-up data (median of at least 3 years between biopsies), progression has been reported in 27-43\% of patients with NASH [5-9]. Cirrhosis was identified in more than one-fifth (22\%) of individuals with NASH included in a large registry study of liver biopsy specimens (mean follow-up of 8.2 years) [10]. NASH is often associated with type 2 diabetes mellitus (T2DM) [11-13], central or visceral obesity [14], dyslipidaemia [15, 16] and hypertension [17]. Together, these conditions comprise the metabolic syndrome [1820]. The pathophysiology of NASH is likely to be multifactorial and may include combinations of metabolic, genetic, environmental and microbial gut factors [21].

In the absence of approved pharmacological therapies, treatment of associated metabolic comorbidities, weight reduction, dietary restrictions and incorporation of an exercise routine remain the cornerstones of management $[22,23]$. Weight loss, however, is difficult to achieve and maintain [24]. Globally, NASH is a disease with a significant unmet medical need, and its incidence is growing at a rate that parallels that of the obesity epidemic [25]. Indeed, in a recent registry study in the US, the incidence of NASH among adults on the waiting list for a liver transplant increased by $170 \%$ between 2004 and 2013 [26]. While this study showed that NASH was the second most common reason for transplantation in 2013 [26], results of a more recent registry analysis indicated that, as of 2014, it had surpassed hepatitis C virus infection as the leading indication for a liver transplant in adults under 50 years of age in the US [27].

Volixibat (SHP626; formerly LUM002) is a highly potent, minimally absorbed, selective inhibitor of the apical sodium-dependent bile acid transporter (ASBT), a transmembrane protein primarily expressed on the luminal surface of ileal enterocytes. ASBT selectively absorbs approximately $60 \%$ of excreted bile acids (BAs) in the lumen of the ileum and is critical for intestinal reabsorption of BAs during enterohepatic recirculation (approximately $95 \%$ of BAs secreted into bile are recycled back to the liver) [28-30].

Enterohepatic circulation of bile acids between the liver and the intestine plays a central role in facilitating the absorption of dietary lipids, and BAs have important regulatory effects on hepatic lipid and glucose metabolism [31]. Several BA signalling pathways in the liver and intestine have been described, and BAs are known to activate specific nuclear receptors, such as the farnesoid $\mathrm{X}$ receptor (FXR), and cell surface receptors, including the $G$ protein-coupled BA receptor TGR5 (also called GPBAR1 or M-BAR) [32, 33]. In the intestine, BAs stimulate the release of peptide hormones, including glucagon-like peptide-1 and -2 (GLP-1 and GLP-2) and peptide YY (PYY), that stimulate insulin secretion [34], inhibit glucagon secretion (glucose dependently) [35], and modulate intestinal growth and function as well as appetite [36]. BAs also stimulate the release of fibroblast growth factors (FGFs), such as FGF-19 and FGF-21 [37], that act in the liver to regulate BA synthesis and glucose and lipid metabolism [31].

Inhibiting ASBT results in a reduction in BA reabsorption, which is associated with an increase in the levels of BAs excreted in the faeces and a decrease in the levels of BAs returning to the liver [38-41]. As assessed by serum levels of $7 \alpha$-hydroxy-4-cholesten-3-one (C4), an intermediate in the biosynthesis of BAs from cholesterol, an upregulation of BA synthesis in the liver is observed following ASBT inhibition [38-42]. BA synthesis uses lowdensity lipoprotein cholesterol (LDL-C) as a precursor, and inhibiting enterohepatic reuptake of BAs has been shown to reduce serum LDL-C levels [43]. The role of BAs in both lipid and glucose metabolism has led to the development of ASBT inhibitors as potential pharmacotherapies for hyperlipidaemia [44] and, subsequently, T2DM and the associated metabolic syndrome [45].

Abnormal cholesterol metabolism and accumulation of free cholesterol in the liver have been implicated in the pathogenesis of NASH [46]. Free cholesterol is directly toxic to hepatocytes and is associated with the inflammation and fibrosis observed in this disease [46]. Preclinical studies of ASBT inhibitors have demonstrated positive effects of these agents in NASHassociated conditions: lowering LDL-C and triglyceride levels in mice [42] and guinea pigs [47] fed high-fat diets, and improving glycaemic control in Zucker diabetic fatty rats $[45,48]$. In mice fed a high-fat diet, treatment with the ASBT inhibitor SC-435 normalised levels 
of hepatic triglycerides and serum cholesterol, significantly improved insulin resistance and decreased the NAFLD histological activity score (predominantly steatosis item scores) [49]. In addition, treatment with the ASBT inhibitor resulted in a shift in the serum BA pool from antagonists of FXR to agonists of FXR [49]. Removal of cholesterol from the liver is a potential treatment approach that could decrease, and possibly reverse, damage to hepatocytes. It is postulated that reducing the levels of BAs returning to the liver via inhibition of ASBTs in the terminal ileum may stimulate the liver to synthesise new BA from cholesterol in the liver and serum, and may have therapeutically beneficial metabolic, anti-inflammatory, anti-steatotic and anti-fibrotic effects in patients with NASH [49].

This paper reports the results of a phase 1, placebocontrolled, dose-escalation study of the ASBT inhibitor volixibat in healthy adults and patients with T2DM. The objectives of the study were to evaluate the safety and tolerability, and the pharmacodynamic (PD) and pharmacokinetic (PK) effects, of multiple doses of volixibat administered orally over a 28 -day period.

\section{Methods}

\section{Study design}

This was a 28-day, randomised, double-blind (investigators and participants), parallel-group study in healthy volunteers (HVs) and patients with T2DM. For HVs, screening took place from day -21 to day -1 and for patients with T2DM from day -42 to day -16 . Patients with T2DM discontinued their anti-diabetic medications starting on day -14 . Thereafter, these individuals measured glucose levels once daily, after an overnight fast, up to day 28 to check that their fasting blood glucose levels were below $12.5 \mathrm{mmol} / \mathrm{L}(225 \mathrm{mg} / \mathrm{dL})$. Patients with T2DM were withdrawn from the study if their fasting blood glucose level reached $12.5 \mathrm{mmol} / \mathrm{L}$ on two consecutive days during the treatment period.

Participants were randomised 3:1 to receive once-daily oral doses of volixibat $(0.5 \mathrm{mg}, 1.0 \mathrm{mg}, 5.0 \mathrm{mg}$ or $10.0 \mathrm{mg})$ or placebo for 28 days. The group of participants assigned to receive the lowest dose of volixibat (or placebo) in the HV cohort commenced treatment first. Further groups of HVs were started on escalating doses (or placebo) at 2week intervals (Fig. 1), contingent upon acceptable assessments of safety and tolerability from the preceding dose. In the T2DM cohort, patients began treatment with volixibat $10 \mathrm{mg}$ (or placebo) 2 weeks after the HV cohort. Clinic visit schedules are shown in Fig. 1. A follow-up telephone call was made on day 56 ( \pm 3 days).

The study was conducted in compliance with the International Conference on Harmonisation E6 Guideline for Good Clinical Practice (Committee for Proprietary Medicinal Products guideline CPMP/ICH/135/95) and was compliant with the European Union Clinical Trial Directive 2001/20/EC. The clinical study protocol, protocol amendments and informed consent forms were reviewed and approved by an independent ethics committee (Stichting Beoordeling Ethiek Biomedisch Onderzoek, Assen, Netherlands). Participants signed the informed consent forms during the pre-study screening visit, within a period of 3 weeks (HVs) or 6 weeks (patients with T2DM) before the first dosing day and before the start of any study-related procedures. Individuals were screened for eligibility by a contract research organisation (Pharmaceutical Research Associates [PRA] Group BV, Zuidlaren, Netherlands); healthy individuals and patients with T2DM were screened within a period of 3 or 6 weeks, respectively, before first administration of the study drug. The randomisation list was prepared by a statistician at PRA who was not involved in the study; within each dose group, a block size of 12 was used to attain a 3:1 randomisation to volixibat or placebo, respectively. Randomisation codes were provided to a pharmacist at PRA who prepared the medication (capsules containing volixibat or placebo were indistinguishable). The study took place between 21 May 2013 and 8 February 2014 at a single study centre in Groningen, Netherlands, and was registered with the Dutch clinical trial authority (Centrale Commissie Mensgebonden Onderzoek; registration number NL44732.056.13).

\section{Participants}

Individuals eligible to participate in the study were men and women aged $18-70$ years (for HVs: $18-55$ years inclusive for doses of $0.5 \mathrm{mg}, 1 \mathrm{mg}$ and $5 \mathrm{mg}$, and $45-$ 65 years inclusive for the $10 \mathrm{mg}$ dose; for patients with T2DM: 18-70 years inclusive) with a body mass index (BMI) of $18.0-30.0 \mathrm{~kg} / \mathrm{m}^{2}$ for HVs and $22.0-35.0 \mathrm{~kg} / \mathrm{m}^{2}$ for patients with T2DM.

Patients with T2DM had to have a glycosylated haemoglobin $\left(\mathrm{HbA}_{1 \mathrm{c}}\right)$ level greater than $6.0 \%$ and less than $10 \%$ at screening, and a fasting blood glucose level of 7.0$12.5 \mathrm{mmol} / \mathrm{L}(126-225 \mathrm{mg} / \mathrm{dL})$ at entry into the clinical research centre (day -2 ). Patients with T2DM were also required to have been taking a stable dose of one or more oral anti-diabetic medications (e.g. metformin, sulphonylurea or any other orally administered glucose-lowering drug) for at least 3 months before screening, to have been receiving no other medications, including dietary supplements, which significantly alter blood glucose control, and to be able and willing to wash out all anti-diabetic medication for 14 days before dosing.

HVs were excluded from the study if they had a history of chronic disease. Patients with T2DM were excluded if they had used insulin or thiazolidinediones 3 months before screening, or if they had advanced diabetic complications, including neuropathy, nephropathy 


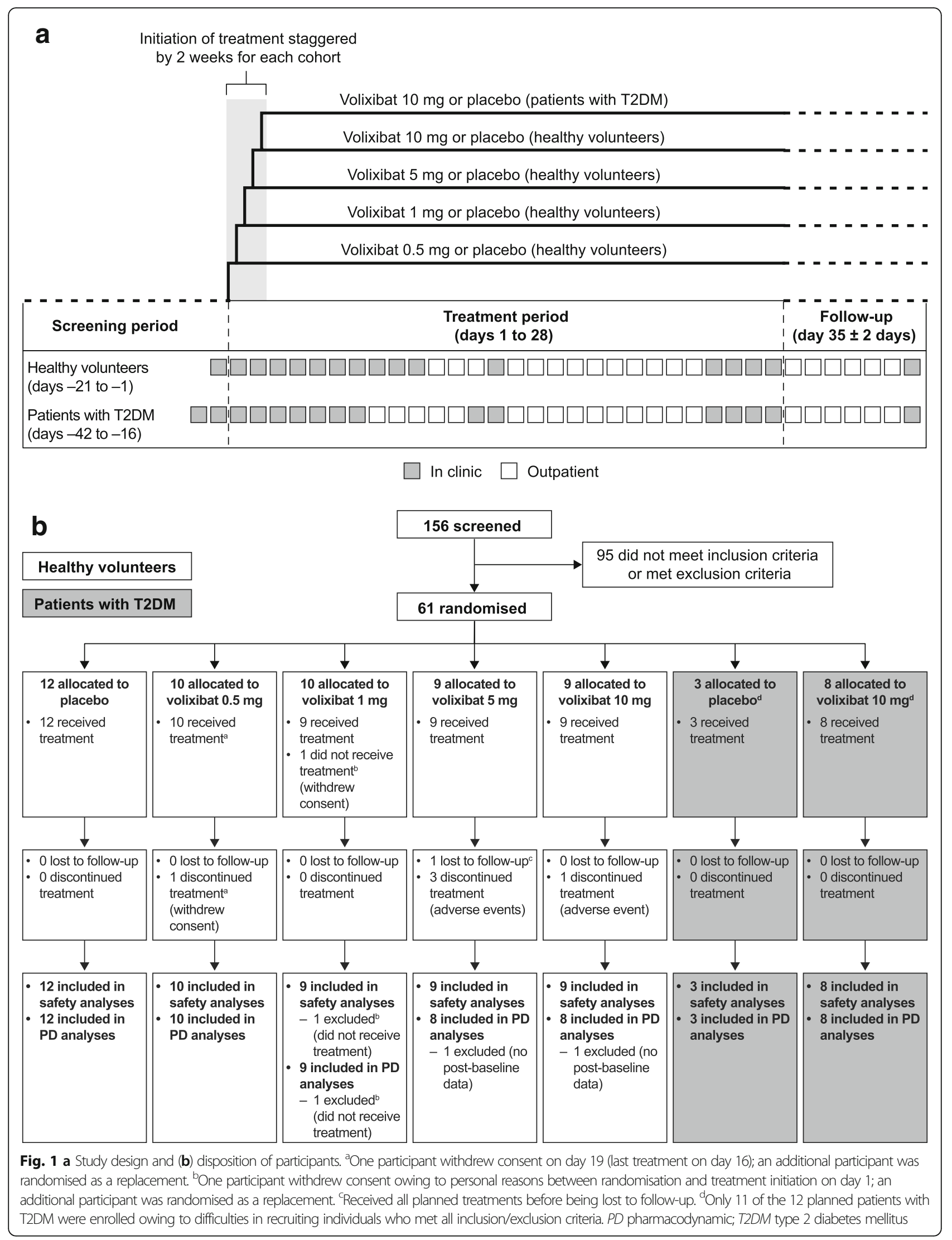


and retinopathy. Complete inclusion and exclusion criteria are provided in Additional file 1.

\section{Outcome measures}

The primary objective of this study was to evaluate the safety and tolerability of orally administered volixibat in adult HVs and patients with T2DM. Safety assessments were based on recording of treatment-emergent adverse events (TEAEs), vital signs, 12-lead electrocardiogram analyses, results of clinical laboratory tests, physical examinations and, in the T2DM cohort, blood glucose measurements.

Secondary objectives were to evaluate the PK and PD properties of volixibat, to explore the effect of volixibat on liver enzymes (alanine aminotransferase, aspartate transaminase, $\gamma$-glutamyl transferase, alkaline phosphatase and lactate dehydrogenase) and fat absorption parameters (levels of 25-hydroxy vitamin D, $\beta$-carotene, retinol, retinol binding protein, tocopherol and total lipids) and to determine the effect of volixibat on glucose metabolism in patients with T2DM.

\section{PK assessments}

The study aimed to calculate the following PK parameters of volixibat: maximum observed plasma concentration; time of maximum observed plasma concentration; and area under the plasma concentration-time curve (AUC) from time 0 to time $t$, where time $t$ is the time of the last quantifiable plasma concentration.

\section{PD assessments}

In the $\mathrm{HV}$ cohort, the following PD variables were measured: serum and faecal total BA concentrations (amount excreted per $24 \mathrm{~h}$ collection interval), biomarkers of BA synthesis/metabolism [33] (C4, FGF-19 and FGF21 ) and lipids (total cholesterol, LDL-C, high-density lipoprotein cholesterol [HDL-C] and triglycerides). In the T2DM cohort, the following additional PD variables were measured: glucose metabolism biomarkers (levels of fasting glucose, insulin, $\mathrm{HbA}_{1 \mathrm{c}}$ and fructosamine) and meal tolerance test (MTT) response (concentrations of glucose, insulin, C-peptide, total GLP-1, GLP-2 and PYY).

\section{Data analysis}

A placebo arm was included in the study design to investigate whether any observed abnormalities were likely to be due to active treatment with volixibat or to the study procedures, and not for a formal comparison between the volixibat and placebo groups. The sample size was based on previous experience and was selected to ensure that safety and tolerability could be assessed adequately while minimising unnecessary exposure to volixibat. On this basis, the study planned to have 12 individuals (volixibat, $n=9$; placebo, $n=3$ ) in each of four groups of HVs and in one group of patients with T2DM, giving a total planned sample size of 60 participants.

All participants who had received at least one dose of study drug and had at least one post-baseline PD assessment were included in PD analyses. PK analyses were carried out on data from all participants who had received volixibat and for whom sufficient bioanalytical data were available with which to calculate reliable estimates of PK parameters. Safety analyses were carried out on data from all participants who had received at least one dose of the study drug.

Statistical analyses were performed using $\mathrm{SAS}^{\circ}$ Version 9.1.3 (SAS Institute Inc., Cary, NC, USA). Safety, PK and PD data were summarised by volixibat dose within each cohort, and safety and PD data were summarised for patients receiving placebo within each cohort using descriptive statistics (number of observations, mean, standard deviation $[\mathrm{SD}]$, median, and minimum and maximum values). Categorical data were summarised using frequencies and percentages. For assessment of changes in parameters from baseline, the baseline was taken as the last measurement before the first dose of the study drug; for assessment of changes in glucose parameters from baseline, the baseline was taken as the pre-MTT measurement. Levels of glucose metabolism biomarkers (glucose, insulin, C-peptide, total GLP-1, GLP-2 and PYY) in the T2DM cohort were compared on days 14 and 28 in the volixibat $10 \mathrm{mg}$ and placebo groups in an exploratory analysis of the following PD parameters: pre-MTT concentration $\left(\mathrm{C}_{\mathrm{pre}}\right)$; maximum observed change from the pre-MTT baseline measurement $\left(E_{\max }\right)$; AUC from time 0 to $3 \mathrm{~h}$ after the MTT $\left(\mathrm{AUC}_{(0-}\right.$ 3) ) calculated using the linear trapezoidal rule; AUC from time 0 to $3 \mathrm{~h}$ after the MTT with the baseline value subtracted $\left(\mathrm{rAUC}_{(0-3)}\right)$ and calculated using the linear trapezoidal rule as $\mathrm{rAUC}_{(0-3)}=\mathrm{AUC}_{(0-3)}-\left(\mathrm{C}_{\text {pre }} \times 3\right)$; updated homeostasis model assessment of insulin resistance (HOMA2-IR) [50]; and homeostasis model assessment of $\beta$-cell function (HOMA2-\%B). These comparisons were performed using a mixed model with treatment, study day and treatment-by-study-day interaction as fixed factors, participant within treatment as a random factor and the day -1 (baseline) value for each participant as a covariate. From this model, the difference in each PD parameter, 90\% confidence intervals (CIs) and $P$ values were calculated on days 14 and 28. As these exploratory comparisons were not corrected for multiplicity, all $P$ values should be considered to be nominal.

\section{Results}

Participant disposition and demographics

During dose escalation, the safety and tolerability of volixibat were considered to be acceptable at each planned dose level by the principal investigator and the independent 
ethics committee. Therefore, participants were assigned to receive volixibat up to the maximum planned daily dose of $10 \mathrm{mg}$. Of the 61 individuals who were randomised, 60 received at least one dose of study drug ( $49 \mathrm{HVs}$ and 11 patients with T2DM) and were included in the safety population. The PD population included 58 of these participants because two individuals did not have a postbaseline PD assessment. Details are provided in Fig. 1b.

Seven participants discontinued from the study (Fig. 1b): four were withdrawn from the study owing to TEAEs (of which one was a serious TEAE, but this was not considered to be treatment-related), two withdrew consent, and one was lost to follow-up. One HV in the volixibat $0.5 \mathrm{mg}$ group and one in the volixibat $1 \mathrm{mg}$ group who withdrew were replaced with additional participants. Owing to the difficulty in recruiting patients with T2DM who met all the inclusion criteria, only 11 of the 12 participants planned for enrolment in this cohort were randomised (volixibat, $n=8$; placebo, $n=3$ ). In total, 54 participants completed the study according to the protocol.

Baseline participant demographics are summarised in Table 1. Within the HV cohort, variations in age among the five groups were consistent with variations in the age enrolment criteria for each group. The ratio of men to women was approximately 1:1 in the HV cohort, except for in the volixibat $0.5 \mathrm{mg}$ and $5 \mathrm{mg}$ dose groups, in which $20.0 \%$ and $22.2 \%$, respectively, were women. There was minimal variation in BMI among members of the HV dose groups, and the majority of participants were Caucasian. All patients in the T2DM cohort were men, and all but one participant were Caucasian. In the T2DM cohort, there were no notable differences in baseline characteristics between the volixibat and placebo groups.

\section{PK effects}

PK parameters could not be calculated because serum concentrations of volixibat were below the lower limit of quantitation $(0.05 \mathrm{ng} / \mathrm{mL})$ at all time points in all but one study sample; a patient in the volixibat $10 \mathrm{mg}$ group of the T2DM cohort had a plasma concentration of $0.06 \mathrm{ng} / \mathrm{mL}$ at $147 \mathrm{~h}$ post-dose.

\section{Safety and tolerability}

Of the 60 participants included in the safety population, study medication was received by $49 \mathrm{HVs}$ (volixibat, $n=37$; placebo, $n=12$ ) and 11 patients with T2DM (volixibat, $n=8$; placebo, $n=3$ ). Dosing for 28 consecutive days was achieved in 55 participants.

\section{TEAEs}

In total, 240 TEAEs were reported in 43 HVs (87.8\%), and 24 TEAEs were reported in 8 patients with T2DM (72.7\%) (Table 2). Most TEAEs were mild (HVs, $n=214$; patients with T2DM, $n=17$ ) or moderate (HVs, $n=26$; patients with T2DM, $n=7$ ) in severity, and no deaths occurred. There was no clear relationship between the treatment groups in the frequency of moderate TEAEs.

One participant in the HV cohort (10 mg dose group) was withdrawn from the study owing to a serious TEAE (ablation of the retina with a bleed in the vitreous body of the right eye), which was considered by the principal investigator to be unrelated to the study drug. Three participants were withdrawn for non-serious TEAEs. In one of these participants, the TEAE was not considered to be treatment-related (moderate Epstein-Barr virus [EBV] infection; HV cohort, $5 \mathrm{mg}$ dose group). The treatment-related TEAEs were mild diarrhoea accompanied by blood in one individual, and mild diarrhoea and mild anal erosion in another individual (both in the HV cohort, $5 \mathrm{mg}$ dose group). The TEAE of mild diarrhoea accompanied by blood was first reported on day 3 after the third dose of volixibat and resolved without medication 3 days after treatment discontinuation on day 5 . In the participant with mild diarrhoea and mild anal erosion, diarrhoea was first reported on day 1 and continued until the patient was withdrawn on day 21;

Table 1 Demographic and baseline characteristics

\begin{tabular}{|c|c|c|c|c|c|c|c|}
\hline \multirow[t]{3}{*}{ Characteristic } & \multicolumn{5}{|c|}{ Healthy volunteers } & \multicolumn{2}{|c|}{ Patients with T2DM } \\
\hline & \multirow{2}{*}{$\begin{array}{l}\text { Placebo } \\
(n=12)\end{array}$} & \multicolumn{4}{|l|}{ Volixibat } & \multirow{2}{*}{$\begin{array}{l}\text { Placebo } \\
(n=3)\end{array}$} & \multirow{2}{*}{$\begin{array}{l}\text { Volixibat } \\
10 \mathrm{mg} \\
(n=8)\end{array}$} \\
\hline & & $\begin{array}{l}0.5 \mathrm{mg} \\
(n=10)\end{array}$ & $\begin{array}{l}1 \mathrm{mg} \\
(n=9)\end{array}$ & $\begin{array}{l}5 \mathrm{mg} \\
(n=9)\end{array}$ & $\begin{array}{l}10 \mathrm{mg} \\
(n=9)\end{array}$ & & \\
\hline Age, years & $\begin{array}{l}38.9 \pm 15.58 \\
(20-62)\end{array}$ & $\begin{array}{l}28.2 \pm 11.65 \\
(19-54)\end{array}$ & $\begin{array}{l}28.9 \pm 10.86 \\
(19-45)\end{array}$ & $\begin{array}{l}30.2 \pm 12.46 \\
(19-54)\end{array}$ & $\begin{array}{l}59.6 \pm 4.72 \\
(55-65)\end{array}$ & $\begin{array}{l}67.7 \pm 2.08 \\
(66-70)\end{array}$ & $\begin{array}{l}65.5 \pm 3.38 \\
(61-70)\end{array}$ \\
\hline $\mathrm{BMI}, \mathrm{kg} / \mathrm{m}^{2}$ & $\begin{array}{l}23.82 \pm 1.816 \\
(21.6-27.4)\end{array}$ & $\begin{array}{l}23.74 \pm 3.301 \\
(20.1-28.7)\end{array}$ & $\begin{array}{l}24.10 \pm 2.841 \\
(20.2-28.9)\end{array}$ & $\begin{array}{l}23.12 \pm 3.423 \\
(19.0-29.1)\end{array}$ & $\begin{array}{l}22.79 \pm 2.958 \\
(18.7-28.8)\end{array}$ & $\begin{array}{l}29.77 \pm 1.914 \\
(28.0-31.8)\end{array}$ & $\begin{array}{l}29.49 \pm 3.512 \\
(24.4-34.8)\end{array}$ \\
\hline Female, $n(\%)$ & $7(58.3)$ & $2(20.0)$ & $4(44.4)$ & $2(22.2)$ & $5(55.6)$ & 0 & 0 \\
\hline \multicolumn{8}{|l|}{ Race, $n(\%)$} \\
\hline Caucasian & $11(91.7)$ & $9(90.0)$ & $7(77.8)$ & $7(77.8)$ & $9(100.0)$ & $3(100.0)$ & $7(87.5)$ \\
\hline Other & $1(8.3)$ & $1(10.0)$ & $2(22.2)$ & $2(22.2)$ & 0 & 0 & 1 (12.5) \\
\hline
\end{tabular}

BMI body mass index, T2DM type 2 diabetes mellitus

Values are mean \pm standard deviation (range) unless otherwise stated; data are from the safety analysis set 
Table 2 Summary of treatment-emergent adverse events

\begin{tabular}{|c|c|c|c|c|c|c|c|}
\hline \multirow[t]{3}{*}{ Participants, $n(\%)$} & \multicolumn{5}{|c|}{ Healthy volunteers } & \multicolumn{2}{|c|}{ Patients with T2DM } \\
\hline & \multirow[b]{2}{*}{$\begin{array}{l}\text { Placebo } \\
(n=12)\end{array}$} & \multicolumn{4}{|l|}{ Volixibat } & \multirow{2}{*}{$\begin{array}{l}\text { Placebo } \\
(n=3)\end{array}$} & \multirow{2}{*}{$\begin{array}{l}\text { Volixibat } \\
10 \mathrm{mg} \\
(n=8)\end{array}$} \\
\hline & & $\begin{array}{l}0.5 \mathrm{mg} \\
(n=10)\end{array}$ & $\begin{array}{l}1 \mathrm{mg} \\
(n=9)\end{array}$ & $\begin{array}{l}5 \mathrm{mg} \\
(n=9)\end{array}$ & $\begin{array}{l}10 \mathrm{mg} \\
(n=9)\end{array}$ & & \\
\hline Any adverse event & $10(83.3)$ & $7(70.0)$ & $9(100)$ & $8(88.9)$ & $9(100)$ & $1(33.3)$ & $7(87.5)$ \\
\hline Treatment-related adverse events & $6(50.0)$ & $7(70.0)$ & $8(88.9)$ & $8(88.9)$ & $9(100)$ & $1(33.3)$ & $6(75.0)$ \\
\hline Severe adverse events & 0 & 0 & 0 & 0 & 0 & 0 & 0 \\
\hline Serious adverse events & 0 & 0 & 0 & 0 & $1(11.1)^{a}$ & 0 & 0 \\
\hline Deaths & 0 & 0 & 0 & 0 & 0 & 0 & 0 \\
\hline \multicolumn{8}{|c|}{ Adverse events reported in $>1$ participant in any group ${ }^{b}$} \\
\hline \multicolumn{8}{|l|}{ Gastrointestinal disorders } \\
\hline Diarrhoea & $3(25.0)$ & $5(50.0)$ & $8(88.9)$ & $8(88.9)$ & $9(100)$ & $1(33.3)$ & $6(75.0)$ \\
\hline Abdominal pain & $3(25.0)$ & $3(30.0)$ & $5(55.6)$ & $6(66.7)$ & $7(77.8)$ & $1(33.3)$ & $3(37.5)$ \\
\hline Gastrointestinal sounds abnormal & $2(16.7)$ & 0 & 0 & $2(22.2)$ & $4(44.4)$ & 0 & $1(12.5)$ \\
\hline Nausea & $2(16.7)$ & $1(10.0)$ & $1(11.1)$ & $1(11.1)$ & $4(44.4)$ & 0 & 0 \\
\hline Flatulence & 0 & $1(10.0)$ & $2(22.2)$ & 0 & $4(44.4)$ & $1(33.3)$ & 0 \\
\hline Abdominal distension & $1(8.3)$ & $2(20.0)$ & $1(11.1)$ & 0 & $2(22.2)$ & 0 & 0 \\
\hline Abdominal discomfort & $1(8.3)$ & $1(10.0)$ & $2(22.2)$ & 0 & 0 & 0 & 0 \\
\hline Dry mouth & 0 & 0 & 0 & 0 & 0 & 0 & $2(25.0)$ \\
\hline \multicolumn{8}{|l|}{ Nervous system disorders } \\
\hline Headache & $4(33.3)$ & $2(20.0)$ & $3(33.3)$ & $1(11.1)$ & $3(33.3)$ & 0 & $1(12.5)$ \\
\hline Dizziness & $1(8.3)$ & 0 & 0 & 0 & $2(22.2)$ & 0 & $1(12.5)$ \\
\hline \multicolumn{8}{|c|}{ General disorders and administration site conditions } \\
\hline Fatigue & $1(8.3)$ & 0 & $2(22.2)$ & $1(11.1)$ & 0 & 0 & 0 \\
\hline Influenza-like illness & 0 & 0 & 0 & $2(22.2)$ & 0 & 0 & 0 \\
\hline \multicolumn{8}{|l|}{ Infections and infestations } \\
\hline Rhinitis & $2(16.7)$ & $1(10.0)$ & 0 & $1(11.1)$ & 0 & 0 & $1(12.5)$ \\
\hline \multicolumn{8}{|c|}{ Respiratory, thoracic and mediastinal disorders } \\
\hline Oropharyngeal pain & $3(25.0)$ & 0 & $1(11.1)$ & 0 & 0 & 0 & 0 \\
\hline \multicolumn{8}{|c|}{ Musculoskeletal and connective tissue disorders } \\
\hline Pain in extremity & 0 & 0 & $2(22.2)$ & 0 & 0 & 0 & 0 \\
\hline
\end{tabular}

T2DM type 2 diabetes mellitus

Data are from the safety analysis set

${ }^{a}$ Ablation of the retina with a bleed in the vitreous body of the right eye (moderate severity), which was not considered to be related to the study drug by the principal investigator. ${ }^{\mathrm{b}}$ Reported by System Organ Class in descending order of frequency in the volixibat 10 mg group for healthy volunteers

anal erosion was first reported on day 13 and persisted for approximately $1.5 \mathrm{~h}$ (the participant reported that they had seen blood on the stools, which came from the anal erosion due to the diarrhoea).

Most TEAEs were transient and resolved before the end of the study; one TEAE of mild oropharyngeal pain was ongoing at follow-up, and three TEAEs were improving. TEAEs considered to be related to the study drug were reported in $38 \mathrm{HVs}(77.6 \%)$ and 7 patients with T2DM (63.6\%); the majority of drugrelated TEAEs were categorised as gastrointestinal disorders, which included diarrhoea and abdominal pain (Table 2).

\section{Laboratory tests and clinical examinations}

With the exception of the participant who developed EBV infection, there were no clinically relevant variations in vital signs. Physical examinations did not identify any abnormalities of clinical relevance. No trends or clinically significant changes in heart rate or rhythm or body weight were observed. In the T2DM cohort, there were no abnormalities in fasting blood glucose levels. Although several individual changes from baseline were observed in the clinical laboratory values, no clinically relevant changes were seen, including those relating to levels of serum electrolytes and serum liver enzymes, and fat absorption parameters. 


\section{PD effects}

\section{$B A$ uptake and synthesis}

Mean total faecal BA excretion was approximately 1.6-3.2 times higher in HVs who received once-daily volixibat for 28 days than in those receiving placebo (Fig. 2a). Mean total BA excretion $( \pm \mathrm{SD})$ was greatest with volixibat $10 \mathrm{mg}(1239.3 \pm 613.42 \mu \mathrm{mol} / 24 \mathrm{~h}$ compared with 386.93 $\pm 413.56 \mu \mathrm{mol} / 24 \mathrm{~h}$ in the placebo group); no clear relationship between BA excretion and dose was discernible at doses of $0.5-5 \mathrm{mg}$. In the T2DM cohort, the mean total BA excretion on day 28 was approximately eight times higher with volixibat $10 \mathrm{mg}(1786.0 \pm 1138.1 \mu \mathrm{mol} / 24 \mathrm{~h})$ than with placebo $(220.0 \pm 259.08 \mathrm{~mol} / 24 \mathrm{~h})$.

At baseline (day 1), there were no notable differences in fasting serum concentrations of BAs (total), FGF-19 or FGF-21 between the volixibat and placebo groups in either the HV or T2DM cohorts (Table 3). No notable changes from baseline to day 28 in these serum a

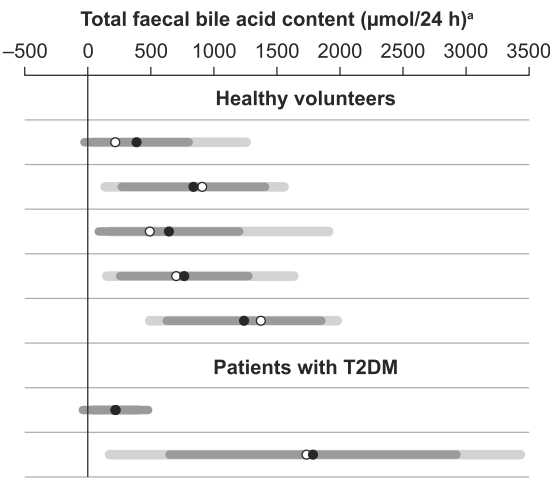

b

Change in total serum bile acid concentration ( $\mu \mathrm{mol} / \mathrm{L}$ ) $-20$

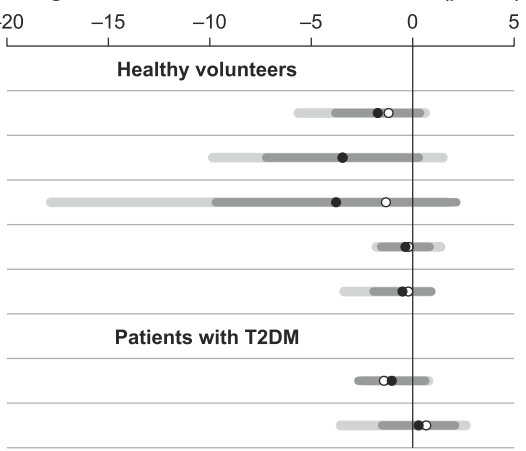

C

Change in serum $\mathrm{C} 4$ concentration $(\mathrm{ng} / \mathrm{mL})$

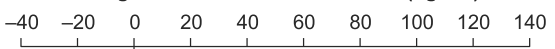

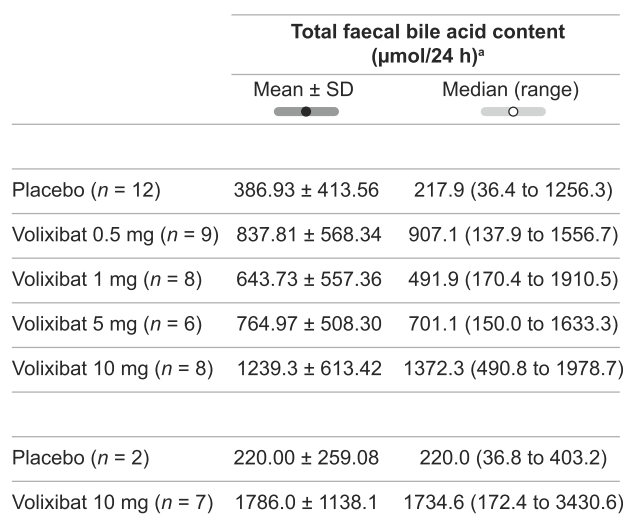

Change in total serum bile acid concentration $(\mu \mathrm{mol} / \mathrm{L})$

Mean \pm SD Median (range) (M)

\begin{tabular}{lcc} 
& Mean \pm SD & Median (range) \\
\hline Placebo $(n=12)$ & $-1.725 \pm 2.0894$ & $-1.19(-5.64$ to 0.62$)$ \\
\hline Volixibat $0.5 \mathrm{mg}(n=9)$ & $-3.462 \pm 3.7605$ & $-3.45(-9.86$ to 1.49$)$ \\
\hline Volixibat $1 \mathrm{mg}(n=9)$ & $-3.777 \pm 5.9272$ & $-1.32(-17.84$ to 1.74$)$ \\
\hline Volixibat $5 \mathrm{mg}(n=6)$ & $-0.360 \pm 1.1948$ & $-0.20(-1.79$ to 1.38$)$ \\
\hline Volixibat $10 \mathrm{mg}(n=8)$ & $-0.505 \pm 1.4245$ & $-0.22(-3.38$ to 0.88$)$ \\
\hline & & \\
\hline Placebo $(n=3)$ & $-1.030 \pm 1.6499$ & $-1.42(-2.45$ to 0.78$)$ \\
\hline Volixibat $10 \mathrm{mg}(n=8)$ & $0.291 \pm 1.8009$ & $0.67(-3.56$ to 2.62$)$
\end{tabular}

Change in serum $\mathrm{C} 4$ concentration $(\mathrm{ng} / \mathrm{mL})$
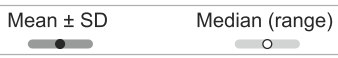

\begin{tabular}{lcc}
\hline Placebo $(n=12)$ & $-14.72 \pm 9.519$ & $-14.5(-30.2$ to -0.4$)$ \\
\hline Volixibat $0.5 \mathrm{mg}(n=9)$ & $7.21 \pm 17.148$ & $4.0(-15.8$ to 41.4$)$ \\
\hline Volixibat $1 \mathrm{mg}(n=9)$ & $10.43 \pm 11.434$ & $5.8(-0.4$ to 30.2$)$ \\
\hline Volixibat $5 \mathrm{mg}(n=6)$ & $24.07 \pm 24.090$ & $12.8(4.5$ to 65.6$)$ \\
\hline Volixibat $10 \mathrm{mg}(n=8)$ & $59.11 \pm 35.927$ & $42.5(22.6$ to 115.2$)$ \\
\hline & & \\
\hline Placebo $(n=3)$ & $12.13 \pm 9.143$ & $15.0(1.9$ to 19.5$)$ \\
\hline Volixibat $10 \mathrm{mg}(n=8)$ & $31.66 \pm 25.426$ & $33.4(-1.7$ to 61.3$)$
\end{tabular}

Fig. 2 Bile acid uptake and synthesis: (a) total faecal bile acid content on day 28 and change from baseline to day 28 in (b) total serum bile acid and (c) C4 concentration. $n$ is the number of participants with available data on day 28. Data are from the pharmacodynamic analysis set. ${ }^{a}$ Determined over $48 \mathrm{~h}$ between days 26 and 28. C4 7a-hydroxy-4-cholesten-3-one; SD standard deviation; T2DM type 2 diabetes 
Table 3 Summary of serum bile acids and serum markers of bile acid synthesis/metabolism

\begin{tabular}{|c|c|c|c|c|c|c|c|c|c|c|}
\hline \multirow{3}{*}{ Healthy volunteers } & \multirow[t]{2}{*}{ Day } & \multirow[t]{2}{*}{ Number } & \multicolumn{2}{|c|}{$\underline{\text { Serum bile acid }(\mu \mathrm{mol} / \mathrm{L})}$} & \multicolumn{2}{|c|}{$\mathrm{C} 4$ (ng/mL) } & \multicolumn{2}{|c|}{ FGF-19 (pg/mL) } & \multicolumn{2}{|c|}{ FGF-21 (pg/mL) } \\
\hline & & & Mean & SD & Mean & SD & Mean & SD & Mean & SD \\
\hline & & & & & & & & & & \\
\hline \multirow[t]{3}{*}{ Placebo } & 1 & 12 & 4.676 & 3.3066 & 29.95 & 12.917 & 359.2 & 500.55 & 174.1 & 90.84 \\
\hline & 14 & 12 & 2.941 & 2.2596 & 15.21 & 12.211 & 264.3 & 280.50 & 224.2 & 180.73 \\
\hline & 28 & 12 & 2.951 & 2.3758 & 15.23 & 8.537 & 259.3 & 333.54 & 167.6 & 112.15 \\
\hline \multirow[t]{3}{*}{ Volixibat $0.5 \mathrm{mg}$} & 1 & 10 & 5.886 & 4.2363 & 22.87 & 6.427 & 304.8 & 328.52 & 195.6 & 155.49 \\
\hline & 14 & 10 & 2.304 & 1.6197 & 35.57 & 25.119 & 297.0 & 302.87 & 219.7 & 133.12 \\
\hline & 28 & 9 & 2.540 & 1.9897 & 30.21 & 13.358 & 306.7 & 247.72 & 204.6 & 104.43 \\
\hline \multirow[t]{3}{*}{ Volixibat 1 mg } & 1 & 9 & 5.614 & 5.7738 & 14.49 & 5.304 & 495.3 & 969.34 & 138.9 & 118.64 \\
\hline & 14 & $9^{a}$ & 4.176 & 3.9589 & 23.60 & 13.736 & 551.1 & 1129.55 & 206.6 & 195.40 \\
\hline & 28 & $9^{a}$ & 1.838 & 0.9931 & 24.92 & 14.686 & 1056.7 & 2428.36 & 150.2 & 99.92 \\
\hline \multirow[t]{3}{*}{ Volixibat 5 mg } & 1 & 8 & 2.826 & 1.4719 & 30.78 & 19.365 & 669.0 & 1301.49 & 224.0 & 111.03 \\
\hline & 14 & 8 & 1.861 & 1.4777 & 56.38 & 43.306 & 651.4 & 1324.94 & 252.3 & 146.26 \\
\hline & 28 & 6 & 2.872 & 1.6163 & 56.52 & 38.863 & 784.7 & 1405.55 & 215.2 & 142.22 \\
\hline \multirow[t]{3}{*}{ Volixibat $10 \mathrm{mg}$} & 1 & 8 & 2.248 & 1.7120 & 13.75 & 4.602 & 228.1 & 239.87 & 204.1 & 156.90 \\
\hline & 14 & 8 & 1.959 & 1.1725 & 37.51 & 18.886 & 186.6 & 242.72 & 253.9 & 165.21 \\
\hline & 28 & 8 & 1.743 & 0.9222 & 72.86 & 35.499 & 164.6 & 226.91 & 212.6 & 157.95 \\
\hline \multicolumn{11}{|l|}{ Patients with T2DM } \\
\hline \multirow[t]{3}{*}{ Placebo } & 1 & $3^{b}$ & 2.370 & 1.6532 & 16.60 & 6.899 & 211.5 & 212.84 & 360.0 & 300.66 \\
\hline & 14 & 3 & 2.523 & 2.2756 & 14.40 & 3.568 & 94.3 & 121.25 & 463.0 & 386.47 \\
\hline & 28 & $3^{b}$ & 1.340 & 0.1758 & 28.73 & 8.545 & 173.5 & 197.28 & 309.3 & 184.02 \\
\hline \multirow[t]{3}{*}{ Volixibat $10 \mathrm{mg}$} & 1 & 8 & 1.826 & 1.6175 & 30.15 & 14.351 & 605.8 & 1264.78 & 334.0 & 128.76 \\
\hline & 14 & $8^{c}$ & 2.355 & 1.1042 & 59.66 & 28.221 & 640.1 & 1399.18 & 497.9 & 198.04 \\
\hline & 28 & 8 & 2.118 & 0.9359 & 61.81 & 22.163 & 114.4 & 146.91 & 359.0 & 178.13 \\
\hline
\end{tabular}

C4 7a-hydroxy-4-cholesten-3-one, FGF fibroblast growth factor, $N$ number of patients for whom data were available, $S D$ standard deviation, T2DM type 2 diabetes mellitus On day 1 (baseline), measurements were taken before participants received the first dose of study drug. Data are from the pharmacodynamic analysis set ${ }^{\text {a}}$ Fibroblast growth factor data available for 8 patients on day 14 and 7 patients on day 28 . ${ }^{b}$ Fibroblast growth factor data available for 2 patients on day 1 and 2

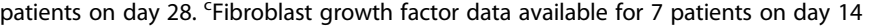

measures were observed, and differences between the volixibat and placebo groups were not considered to be clinically relevant. In the HV cohort, a slight reduction in mean $( \pm$ SD) fasting total serum BA concentration was observed from baseline to day 28 in all groups, which was most pronounced in the volixibat $0.5 \mathrm{mg}(-3.462 \pm$ $3.7605 \mu \mathrm{mol} / \mathrm{L})$ and $1 \mathrm{mg}(-3.777 \pm 5.9272 \mu \mathrm{mol} / \mathrm{L})$ groups (Fig. 2b). No notable dose-related changes were seen in the major serum BA species (cholic acid, chenodeoxycholic acid, deoxycholic acid, ursodeoxycholic acid and lithocholic acid) in the HV or T2DM cohorts.

Serum C4 concentrations at baseline and on days 14 and 28 are shown in Table 3. In the HV cohort, there was a dose-dependent increase in mean serum $\mathrm{C} 4$ concentration from baseline to day 28 in the volixibat groups compared with a decrease in the placebo group (Fig. 2c). At volixibat doses of $0.5-5 \mathrm{mg}$, mean serum C4 levels on day 14 were approximately $1.5-1.8$ times higher than baseline and remained similar on day 28 at approximately $1.3-1.8$ times higher than baseline; in the
$10 \mathrm{mg}$ group, levels were 2.7 times higher than baseline on day 14 and increased further to approximately 5.3 times higher than baseline on day 28 (Table 3). In the placebo group, mean serum C4 levels were half that of baseline on days 14 and 28. In the T2DM cohort, mean $( \pm$ SD) serum C4 levels in the volixibat group were approximately twofold greater on day $14 \quad(59.66 \pm$ $28.221 \mathrm{ng} / \mathrm{mL})$ and day $28(61.81 \pm 22.163 \mathrm{ng} / \mathrm{mL})$ than at baseline $(30.15 \pm 14.351 \mathrm{ng} / \mathrm{mL})$ (Table 3). In the placebo group, mean serum C4 levels at baseline (16.60 \pm $6.899 \mathrm{ng} / \mathrm{mL}$ ) were lower than those in the volixibat group, remained similar on day $14(14.40 \pm 3.568 \mathrm{ng} /$ $\mathrm{mL})$ and had increased slightly on day 28 (28.73 \pm $8.545 \mathrm{ng} / \mathrm{mL}$ ) to a level similar to that observed before treatment in the volixibat group.

\section{Lipid profile}

At baseline, all participants were normolipaemic, and there were no major differences among treatment groups in serum lipid concentrations (total cholesterol, 
LDL-C, HDL-C and triglycerides) (Table 4). No clinically relevant changes in lipid concentrations from baseline to day 14 or day 28 were observed in either cohort, and there were no clinically relevant differences between the volixibat and placebo groups on days 14 or 28 . In the HV cohort, the mean total cholesterol level was slightly lower on day 14 and day 28 than at baseline in the volixibat $0.5 \mathrm{mg}, 1 \mathrm{mg}$ and $10 \mathrm{mg}$ groups (Table 4); in the volixibat $5 \mathrm{mg}$ group, there was little variation throughout the study. Similarly, compared with baseline, the concentration of LDL-C was slightly lower on days 14 and day 28 in the volixibat $1 \mathrm{mg}$ and $10 \mathrm{mg}$ groups; in the $0.5 \mathrm{mg}$ and $5 \mathrm{mg}$ groups, no trend was evident between days 1 and 28. Changes from baseline to days 14 and 28 in total cholesterol, LDL-C and triglyceride levels were not clinically significant, but the changes tended to be smaller in the volixibat groups than in the placebo group; no obvious trend in HDL$\mathrm{C}$ changes from baseline was evident between treatment groups (Table 4).

\section{Glucose metabolism in the T2DM cohort}

In patients with T2DM, serum levels of glucose, insulin and C-peptide, and plasma levels of GLP-1, GLP-2 and PYY were assessed pre-dose (day -1 ) and post-dose (days 14 and 28$)$, in a fasting state $(1 \mathrm{~h}$ and immediately before an MTT on days $-1,14$ and 28) and in response to an MTT (30-min intervals up to $3 \mathrm{~h}$ after the MTT on days $-1,14$ and 28 ).

In the volixibat group, fasting glucose levels before the MTT (pre-MTT) were lower on days 14 and 28 than those at pre-dose (day -1$)$; pre-MTT glucose levels did not change appreciably between pre-dose and post-dose in the placebo group (Fig. 3). Compared with placebo, least-squares (LS) mean fasting glucose concentrations before the MTT $\left(\mathrm{C}_{\mathrm{pre}}\right)$ were nominally significantly lower in the volixibat group on day $14(-1.8 \mathrm{mmol} / \mathrm{L} ; 90 \% \mathrm{CI}:-2.8$, $-0.9 ; P=0.0064)$ and day $28(-1.5 \mathrm{mmol} / \mathrm{L} ; 90 \% \mathrm{CI}:-2.5$, -0.6; $P=0.0163$ ) (Table 5). The glucose response was similar for volixibat and placebo at $0.5-3 \mathrm{~h}$ after the MTT (post-MTT), indicating that this was not altered by once-

Table 4 Summary of blood lipid parameters at baseline and on days 14 and 28

\begin{tabular}{|c|c|c|c|c|c|c|c|c|c|c|}
\hline & \multirow[t]{2}{*}{ Day } & \multirow[t]{2}{*}{ Number } & \multicolumn{2}{|c|}{ Total cholesterol (mmol/L) } & \multicolumn{2}{|c|}{ LDL-C (mmol/L) } & \multicolumn{2}{|c|}{ HDL-C (mmol/L) } & \multicolumn{2}{|c|}{ Triglycerides (mmol/L) } \\
\hline & & & Mean & SD & Mean & SD & Mean & SD & Mean & SD \\
\hline \multicolumn{11}{|l|}{ Healthy volunteers } \\
\hline \multirow[t]{3}{*}{ Placebo } & 1 & 12 & 4.70 & 0.964 & 2.78 & 0.853 & 1.38 & 0.298 & 1.189 & 0.3600 \\
\hline & 14 & 12 & 4.78 & 1.209 & 3.03 & 1.112 & 1.29 & 0.223 & 1.031 & 0.3635 \\
\hline & 28 & 12 & 4.73 & 1.097 & 2.87 & 0.934 & 1.38 & 0.322 & 1.233 & 0.4302 \\
\hline \multirow[t]{3}{*}{ Volixibat 0.5 mg } & 1 & 10 & 4.17 & 0.933 & 2.39 & 0.861 & 1.35 & 0.314 & 0.976 & 0.3523 \\
\hline & 14 & 10 & 4.07 & 0.686 & 2.23 & 0.726 & 1.31 & 0.307 & 1.013 & 0.3370 \\
\hline & 28 & 9 & 3.99 & 0.854 & 2.29 & 0.851 & 1.37 & 0.274 & 0.936 & 0.2013 \\
\hline \multirow[t]{3}{*}{ Volixibat 1 mg } & 1 & 9 & 4.28 & 0.628 & 2.43 & 0.650 & 1.34 & 0.274 & 0.863 & 0.3278 \\
\hline & 14 & 9 & 3.87 & 0.650 & 2.22 & 0.655 & 1.29 & 0.215 & 0.962 & 0.6851 \\
\hline & 28 & 9 & 3.97 & 0.552 & 2.22 & 0.576 & 1.33 & 0.300 & 1.024 & 0.5973 \\
\hline \multirow[t]{3}{*}{ Volixibat 5 mg } & 1 & 8 & 4.53 & 0.865 & 2.81 & 0.781 & 1.25 & 0.227 & 1.476 & 0.6576 \\
\hline & 14 & 8 & 4.21 & 0.825 & 2.50 & 0.725 & 1.23 & 0.276 & 1.294 & 0.7138 \\
\hline & 28 & 6 & 4.58 & 1.102 & 2.77 & 0.967 & 1.33 & 0.350 & 1.522 & 0.5151 \\
\hline \multirow[t]{3}{*}{ Volixibat 10 mg } & 1 & 8 & 5.44 & 0.637 & 3.04 & 0.641 & 1.86 & 0.421 & 0.875 & 0.4316 \\
\hline & 14 & 8 & 4.94 & 0.774 & 2.66 & 0.715 & 1.80 & 0.518 & 0.914 & 0.5547 \\
\hline & 28 & 8 & 4.98 & 0.504 & 2.59 & 0.559 & 1.94 & 0.346 & 1.055 & 0.5934 \\
\hline \multicolumn{11}{|l|}{ Patients with T2DM } \\
\hline \multirow[t]{3}{*}{ Placebo } & 1 & 3 & 4.60 & 0.964 & 2.83 & 0.874 & 1.07 & 0.115 & 2.260 & 1.0817 \\
\hline & 14 & 3 & 4.37 & 0.586 & 2.57 & 0.404 & 1.03 & 0.153 & 2.527 & 1.1007 \\
\hline & 28 & 3 & 5.03 & 1.266 & 2.93 & 0.603 & 1.13 & 0.153 & 3.070 & 2.4333 \\
\hline \multirow[t]{3}{*}{ Volixibat 10 mg } & 1 & 8 & 4.69 & 1.037 & 3.01 & 0.926 & 1.25 & 0.245 & 1.725 & 0.5263 \\
\hline & 14 & 8 & 4.20 & 1.135 & 2.46 & 1.086 & 1.25 & 0.214 & 2.074 & 0.8015 \\
\hline & 28 & 8 & 4.68 & 0.924 & 2.84 & 0.890 & 1.29 & 0.275 & 1.933 & 0.5337 \\
\hline
\end{tabular}

HDL-C high-density lipoprotein cholesterol, $L D L-C$ low-density lipoprotein cholesterol, $N$ number of patients for whom data were available, $S D$ standard deviation, T2DM type 2 diabetes mellitus

On day 1 (baseline), measurements of faecal bile acids and serum markers were taken before participants received the first dose of study drug; data are from the pharmacodynamic analysis set 

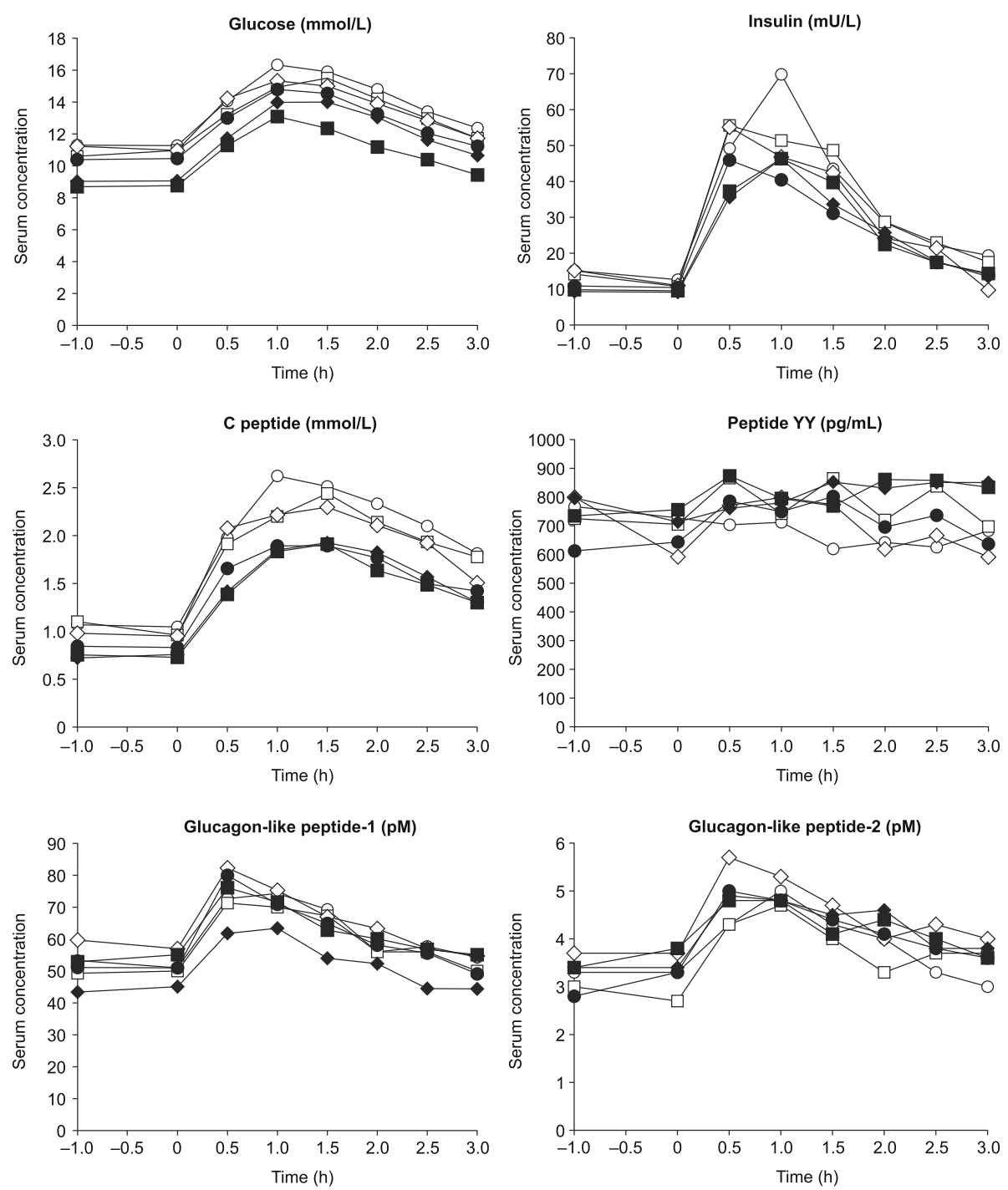

Placebo
$(n=3)$$\left\{\begin{array}{l}-0-\text { Day }-1-- \\ -\square \text { Day 14 }- \\ \checkmark \text { Day 28 }\end{array}\right\} \begin{aligned} & \text { Volixibat } 10 \mathrm{mg} \\ & (n=8)\end{aligned}$

Fig. 3 Arithmetic mean concentration-time profiles (pre-meal tolerance test) for glucose metabolism parameters in patients with type 2 diabetes mellitus. Data are from the pharmacodynamic analysis set

daily volixibat $10 \mathrm{mg}$ administration for 14 or 28 days (Fig. 3). This was confirmed by the $\mathrm{E}_{\max }, \mathrm{AUC}_{(0-3)}$ and rAUC $_{(0-3)}$ values for glucose (Additional file 2: Table S1).

Fasting, pre-MTT insulin and C-peptide levels were similar in the volixibat and placebo groups before dosing (day -1) and after dosing (days 14 and 28). Post-MTT, there were no consistent or nominally significant differences between the volixibat and placebo groups in LS mean $\mathrm{E}_{\text {max }}, \mathrm{AUC}_{(0-3)}$ or $\mathrm{rAUC}_{(0-3)}$ for insulin or $\mathrm{C}$ peptide; for $\mathrm{C}$-peptide, there was a trend towards lower values in the volixibat groups compared with the placebo group (Table 5 and Additional file 2: Table S1). There were no obvious changes in fructosamine or $\mathrm{HbA}_{1 \mathrm{c}}$ levels from baseline to day 28.

For both GLP-1 and GLP-2, pre-MTT levels were similar in the volixibat and placebo groups before dosing (day -1) and after dosing (days 14 and 28) (Fig. 3). There were no nominally significant post-MTT differences between the volixibat and placebo groups in LS mean $\mathrm{E}_{\max }$, $\mathrm{AUC}_{(0-3)}$ or $\mathrm{rAUC}_{(0-3)}$ for GLP-1 at any time point (Table 5). For GLP-2, $E_{\max }$ was nominally significantly lower in the volixibat group than in the placebo group on day $14(-0.99 ; 90 \% \mathrm{CI}:-1.68,-0.30 ; P=0.0280)$, but not on day $28(-0.15 ; 90 \% \mathrm{CI}:-0.84,0.55 ; P=0.7043)$, 
Table 5 Exploratory comparison of glucose metabolism pharmacodynamic parameters between the volixibat $10 \mathrm{mg}$ and placebo groups in patients with type 2 diabetes mellitus

\begin{tabular}{|c|c|c|c|c|c|}
\hline & \multirow[t]{2}{*}{ Parameter } & \multicolumn{2}{|c|}{$\underline{\text { LS mean value }}$} & \multicolumn{2}{|c|}{ Difference in LS mean value (volixibat-placebo) } \\
\hline & & Volixibat & Placebo & Mean $(90 \% \mathrm{Cl})$ & $P$ value \\
\hline \multicolumn{6}{|l|}{ Glucose } \\
\hline \multirow[t]{4}{*}{ Day 14} & $C_{\text {pre }}(\mathrm{mmol} / \mathrm{L})$ & 8.9 & 10.7 & $-1.8(-2.8,-0.9)$ & 0.0064 \\
\hline & $\mathrm{E}_{\max }(\mathrm{mmol} / \mathrm{L})$ & 4.7 & 4.5 & $0.2(-1.8,2.2)$ & 0.8524 \\
\hline & $A \cup C_{(0-3)}(h \cdot m m o l / L)$ & 34.3 & 39.2 & $-4.9(-10.1,0.3)$ & 0.1173 \\
\hline & $\mathrm{rAUC}_{(0-3)}(\mathrm{h} \cdot \mathrm{mmol} / \mathrm{L})$ & 7.6 & 7.5 & $0.1(-4.4,4.6)$ & 0.9739 \\
\hline \multirow[t]{4}{*}{ Day 28} & $C_{\text {pre }}(\mathrm{mmol} / \mathrm{L})$ & 9.2 & 10.7 & $-1.5(-2.5,-0.6)$ & 0.0163 \\
\hline & $\mathrm{E}_{\max }(\mathrm{mmol} / \mathrm{L})$ & 5.6 & 4.4 & $1.1(-0.8,3.1)$ & 0.3147 \\
\hline & $\mathrm{AUC}_{(0-3)}(\mathrm{h} \cdot \mathrm{mmol} / \mathrm{L})$ & 37.8 & 39.6 & $-1.8(-7.0,3.4)$ & 0.5408 \\
\hline & $\mathrm{rAUC}_{(0-3)}(\mathrm{h} \cdot \mathrm{mmol} / \mathrm{L})$ & 10.1 & 7.8 & $2.3(-2.2,6.8)$ & 0.3714 \\
\hline \multicolumn{6}{|l|}{ Insulin } \\
\hline \multirow[t]{4}{*}{ Day 14} & $C_{\text {pre }}(\mathrm{mU} / \mathrm{L})$ & 10.0 & 9.6 & $0.4(-3.3,4.0)$ & 0.8618 \\
\hline & $E_{\max }(m U / L)$ & 41.3 & 50.7 & $-9.4(-26.9,8.1)$ & 0.3509 \\
\hline & $A \cup C_{(0-3)}(h \cdot m U / L)$ & 94.2 & 92.7 & $1.4(-24.1,27.0)$ & 0.9195 \\
\hline & $\operatorname{rAUC}_{(0-3)}(\mathrm{h} \cdot \mathrm{mU} / \mathrm{L})$ & 62.9 & 67.4 & $-4.5(-29.8,20.7)$ & 0.7499 \\
\hline \multirow[t]{4}{*}{ Day 28} & $C_{\text {pre }}(\mathrm{mU} / \mathrm{L})$ & 9.6 & 9.9 & $-0.3(-4.0,3.4)$ & 0.8832 \\
\hline & $\mathrm{E}_{\max }(\mathrm{mU} / \mathrm{L})$ & 42.9 & 39.5 & $3.5(-14.0,20.9)$ & 0.7256 \\
\hline & $A \cup C_{(0-3)}(h \cdot m U / L)$ & 91.8 & 82.4 & $9.4(-16.2,34.9)$ & 0.5173 \\
\hline & $\mathrm{rAUC}_{(0-3)}(\mathrm{h} \cdot \mathrm{mU} / \mathrm{L})$ & 61.8 & 56.4 & $5.4(-19.9,30.6)$ & 0.7045 \\
\hline \multicolumn{6}{|l|}{ C-peptide } \\
\hline \multirow[t]{4}{*}{ Day 14} & $C_{\text {pre }}(\mathrm{nmol} / \mathrm{L})$ & 0.780 & 0.823 & $-0.043(-0.203,0.117)$ & 0.6313 \\
\hline & $E_{\max }(n m o l / L)$ & 1.407 & 1.297 & $0.110(-0.232,0.452)$ & 0.5706 \\
\hline & $\mathrm{AUC}_{(0-3)}(\mathrm{h} . \mathrm{nmol} / \mathrm{L})$ & 5.002 & 4.985 & $0.017(-0.719,0.753)$ & 0.9667 \\
\hline & $\operatorname{rAUC}_{(0-3)}(\mathrm{h} \cdot \mathrm{nmol} / \mathrm{L})$ & 2.631 & 2.601 & $0.030(-0.826,0.886)$ & 0.9500 \\
\hline \multirow[t]{4}{*}{ Day 28} & $C_{\text {pre }}(\mathrm{nmol} / \mathrm{L})$ & 0.810 & 0.812 & $-0.002(-0.162,0.158)$ & 0.9797 \\
\hline & $\mathrm{E}_{\max }(\mathrm{nmol} / \mathrm{L})$ & 1.407 & 1.164 & $0.243(-0.099,0.585)$ & 0.2243 \\
\hline & $A \cup C_{(0-3)}(\mathrm{h} \cdot \mathrm{nmol} / \mathrm{L})$ & 5.181 & 4.930 & $0.251(-0.486,0.987)$ & 0.5443 \\
\hline & $\mathrm{rAUC}_{(0-3)}(\mathrm{h} \cdot \mathrm{nmol} / \mathrm{L})$ & 2.719 & 2.578 & $0.141(-0.715,0.996)$ & 0.7700 \\
\hline \multicolumn{6}{|l|}{ GLP-1 } \\
\hline \multirow[t]{4}{*}{ Day 14} & $C_{\text {pre }}(\mathrm{pM})$ & 55 & 50 & $5(-10,20)$ & 0.5552 \\
\hline & $E_{\max }(p M)$ & 23 & 29 & $-6(-16,4)$ & 0.2731 \\
\hline & $A \cup C_{(0-3)}(h . p M)$ & 191 & 185 & $7(-33,47)$ & 0.7643 \\
\hline & $\operatorname{rAUC}_{(0-3)}(\mathrm{h} . \mathrm{pM})$ & 26 & 35 & $-10(-35,16)$ & 0.5128 \\
\hline \multirow[t]{4}{*}{ Day 28} & $C_{\text {pre }}(\mathrm{pM})$ & 45 & 57 & $-12(-27,3)$ & 0.1894 \\
\hline & $E_{\max }(p M)$ & 21 & 27 & $-6(-16,4)$ & 0.2941 \\
\hline & $A \cup C_{(0-3)}(h . p M)$ & 161 & 200 & $-39(-79,1)$ & 0.1100 \\
\hline & $\operatorname{rAUC}_{(0-3)}$ (h.pM) & 25 & 29 & $-4(-30,21)$ & 0.7645 \\
\hline \multicolumn{6}{|l|}{ GLP-2 } \\
\hline \multirow[t]{4}{*}{ Day 14} & $C_{\text {pre }}(\mathrm{ng} / / \mathrm{mL})$ & 3.80 & 2.72 & $1.08(-0.14,2.31)$ & 0.1396 \\
\hline & $\mathrm{E}_{\max }(\mathrm{ngl} / \mathrm{mL})$ & 1.16 & 2.15 & $-0.99(-1.68,-0.30)$ & 0.0280 \\
\hline & $A \cup C_{(0-3)}(h \cdot n g l / m L)$ & 12.67 & 12.24 & $0.43(-2.06,2.92)$ & 0.7597 \\
\hline & $\operatorname{rAUC}_{(0-3)}(\mathrm{h} \cdot \mathrm{ngl} / \mathrm{mL})$ & 1.46 & 3.59 & $-2.14(-3.89,-0.38)$ & 0.0524 \\
\hline Day 28 & $C_{\text {pre }}(\mathrm{ngl} / \mathrm{mL})$ & 3.40 & 3.84 & $-0.44(-1.66,0.78)$ & 0.5267 \\
\hline
\end{tabular}


Table 5 Exploratory comparison of glucose metabolism pharmacodynamic parameters between the volixibat $10 \mathrm{mg}$ and placebo groups in patients with type 2 diabetes mellitus (Continued)

\begin{tabular}{|c|c|c|c|c|c|}
\hline & \multirow[t]{2}{*}{ Parameter } & \multicolumn{2}{|c|}{ LS mean value } & \multicolumn{2}{|c|}{ Difference in LS mean value (volixibat-placebo) } \\
\hline & & Volixibat & Placebo & Mean $(90 \% \mathrm{Cl})$ & $P$ value \\
\hline & $E_{\max }(n g l / m L)$ & 1.69 & 1.84 & $-0.15(-0.84,0.55)$ & 0.7043 \\
\hline & $A \cup C_{(0-3)}(h \cdot n g l / m L)$ & 12.77 & 14.30 & $-1.53(-4.03,0.96)$ & 0.2886 \\
\hline & $\mathrm{rAUC}_{(0-3)}(\mathrm{h} . \mathrm{ngl} / \mathrm{mL})$ & 2.77 & 2.30 & $0.47(-1.28,2.22)$ & 0.6347 \\
\hline \multicolumn{6}{|l|}{ Peptide YY } \\
\hline \multirow[t]{4}{*}{ Day 14} & $C_{\text {pre }}(p g / m L)$ & 770 & 668 & $102(-173,377)$ & 0.5141 \\
\hline & $E_{\max }(p g / m L)$ & 227 & 270 & $-43(-241,155)$ & 0.6986 \\
\hline & $\mathrm{AUC}_{(0-3)}(\mathrm{h} . \mathrm{pg} / \mathrm{mL})$ & 2444 & 2448 & $-4(-563,555)$ & 0.9897 \\
\hline & $\mathrm{rAUC}_{(0-3)}(\mathrm{h} . \mathrm{pg} / \mathrm{mL})$ & 85 & 578 & $-493(-942,-44)$ & 0.0752 \\
\hline \multirow[t]{4}{*}{ Day 28} & $C_{\text {pre }}(p g / m L)$ & 727 & 555 & $172(-103,447)$ & 0.2808 \\
\hline & $E_{\max }(p g / m L)$ & 249 & 353 & $-105(-302,93)$ & 0.3569 \\
\hline & $A \cup C_{(0-3)}(h \cdot p g / m L)$ & 2398 & 2190 & $208(-351,767)$ & 0.5122 \\
\hline & $r A \cup C_{(0-3)}(h . p g / m L)$ & 167 & 658 & $-491(-941,-42)$ & 0.0760 \\
\hline \multicolumn{6}{|c|}{ Glucose-insulin } \\
\hline \multirow[t]{2}{*}{ Day 14} & HOMA2-\%B (-) & 37.4 & 26.2 & $11.2(0.4,22.0)$ & 0.0888 \\
\hline & HOMA2-IR (-) & 1.5 & 1.5 & $0(-0.6,0.5)$ & 0.9137 \\
\hline \multirow[t]{2}{*}{ Day 28} & HOMA2-\%B (-) & 35.1 & 29.6 & $5.5(-5.3,16.3)$ & 0.3724 \\
\hline & HOMA2-IR (-) & 1.4 & 1.5 & $-0.1(-0.7,0.4)$ & 0.6466 \\
\hline
\end{tabular}

$A U C_{(0-3)}$ area under the effect (serum/plasma concentration)-time curve from time 0 to $3 \mathrm{~h}$ after the MTT calculated using the linear trapezoidal rule, $\mathrm{Cl}$ confidence interval, $C_{\text {pre }}$ pre-MTT concentration, $E_{\max }$ maximum observed change from the pre-MTT baseline measurement, GLP glucagon-like peptide, HOMA2-IR updated homeostasis model assessment of insulin resistance, HOMA2-\%B homeostasis model assessment of $\beta$-cell function, $L S$ mean least-squares mean, $M T T$ meal tolerance test, $r A \cup C_{(0-3)}$ area under the effect (serum/plasma concentration)-time curve from time 0 to $3 \mathrm{~h}$ after the $\mathrm{MTT}$ calculated using the linear trapezoidal rule with baseline subtracted $\left(\mathrm{rAUC}_{(0-3)}=A \cup C_{(0-3)}-\left[C_{\text {pre }} \times 3\right]\right.$ ) (baseline is the value immediately prior to the standardised breakfast [Ensure Plus ${ }^{\oplus}$, Abbott Nutrition, Lake Forest, IL, USA])

Comparisons were made between parameters in the volixibat and placebo groups using a mixed model with treatment, study day and treatment-by-study-day interaction as fixed factors, participant within treatment as a random factor, and the day -1 baseline value for each participant as a covariate; data are from the pharmacodynamic analysis set

and there were no differences in $\mathrm{AUC}_{(0-3)}$ or $\mathrm{rAUC}_{(0-3)}$ on days 14 or 28 .

Fasting, pre-MTT PYY levels were not notably different in the volixibat and placebo groups before dosing (day -1) or after dosing (days 14 and 28) (Fig. 3), and there were no nominally significant post-MTT differences in $\mathrm{C}_{\text {pre }}, \mathrm{E}_{\text {max }}, \mathrm{AUC}_{(0-3)}$ or $\mathrm{rAUC}_{(0-3)}$ at any time point (Table 5 and Additional file 2: Table S1).

In participants receiving volixibat, there was a trend towards an increase in $\beta$-cell function (based on values from HOMA2-\%B) and a decrease in insulin resistance (based on values from HOMA2-IR) on days 14 and 28 compared with pre-treatment (day -1$)$; in the placebo group, HOMA2-\%B and HOMA2-IR values remained relatively constant throughout the study. In the volixibat group, mean $( \pm \mathrm{SD})$ HOMA2-\%B values were higher on day $14(37.00 \pm 7.824)$ and day $28(34.68 \pm 12.161)$ than on day $-1(29.18 \pm 7.177)$ (Additional file 3: Table S2); the difference between the volixibat and placebo groups approached nominal significance on day 14 (difference in LS means: $11.2 ; 90 \% \mathrm{CI}: 0.4,22.0 ; \quad P=0.0888$ ) (Table 5). Conversely, mean HOMA2-IR values were lower on day $14(1.43 \pm 0.483)$ and day $28(1.35 \pm 0.493)$ than on day $-1(1.64 \pm 0.616)$ in the volixibat group (Additional file 3: Table S2); differences between the volixibat and placebo groups were not nominally significant on days 14 or 28 .

\section{Discussion}

In this phase 1 study, inhibition of the ASBT by volixibat at daily doses of $0.5-10.0 \mathrm{mg}$ for 28 days reduced BA reabsorption and substantially increased levels of BA in the colon of HVs and patients with T2DM, as indicated by an increase in faecal BA excretion (a 1.6-3.2-fold increase in HVs and an eightfold increase in patients with T2DM). The elevation in BA excretion was accompanied by increased BA synthesis, as indicated by the rise in serum $\mathrm{C} 4$ concentrations in participants receiving volixibat. If these effects are indicative of removal of free cholesterol from the liver through synthesis of new BA from cholesterol, we hypothesise that larger studies of longer duration involving patients with NASH could result in therapeutically beneficial metabolic, anti-inflammatory, anti-steatotic and anti-fibrotic effects. 
In a recently published congress abstract of a phase 1 study of volixibat in overweight and obese adults [51], volixibat increased faecal BA and serum $\mathrm{C} 4$ levels compared with placebo, indicating that these PD effects are consistent across studies and occur in a patient population that is characteristic of NASH. In this phase 1 study [51], volixibat was administered for 12 days at doses of up to $40 \mathrm{mg}$ daily; doses of at least $20 \mathrm{mg}$ once daily resulted in maximal inhibition of BA reabsorption [51]. In addition, the results of this study demonstrated that the median reduction in total cholesterol level from baseline to the final on-treatment assessment was greater with volixibat than with placebo, and that the median LDL-C level was reduced in all volixibat groups during treatment, compared with a small increase from baseline in the placebo group.

Clinical studies of two other ASBT inhibitors (A4250 and elobixibat; Albireo, Gothenburg, Sweden) have obtained results consistent with those of the current study [38-41, 52]. The authors of a phase 1, placebo-controlled study that included assessment of A4250 treatment for 7 days at doses of $1 \mathrm{mg}$ or $3 \mathrm{mg}$ once daily, or $1.5 \mathrm{mg}$ twice daily, reported that faecal BA excretion was fivefold higher on day 7 in the $3 \mathrm{mg}$ once-daily group than in the placebo group; the magnitude of the treatment effect was less in the $1.5 \mathrm{mg}$ twice-daily group and was similar to that of placebo in the $1 \mathrm{mg}$ once-daily group (no values reported) [52]. The authors also illustrated that plasma $\mathrm{C} 4$ levels increased from baseline to day 7 in the A4250 groups (no values reported) [52]. Four placebo-controlled studies of elobixibat in patients with constipation demonstrated consistent increases in plasma $\mathrm{C} 4$ levels at oncedaily doses of $0.1-20 \mathrm{mg}$ for up to 8 weeks; faecal BA excretion was not assessed in these studies [38-41]. Together with the results of the current study, these findings indicate that ASBT inhibition increases the faecal excretion of BAs and suggest that this may result in upregulation of hepatic BA synthesis, which may help to maintain BA homeostasis in the liver.

Although there was evidence of greater BA synthesis during treatment with volixibat than with placebo in the current study, clinically significant decreases in serum LDL-C levels were not observed. However, in patients with T2DM, those treated with volixibat showed a trend towards increased levels of serum HDL-C, decreased levels of serum triglycerides and reductions in fasting glucose levels, suggesting improvements in both lipid and glucose homeostasis. In the other phase 1 study of volixibat in overweight and obese adults, the median reduction in LDL-C levels over 12 days was $0.6990 \mathrm{mmol} / \mathrm{L}$ across the volixibat groups, which included patients treated with fixed doses of up $40 \mathrm{mg}$ daily [51]. Reductions in LDL-C levels were also observed in three [38-40] of the four studies of elobixibat in patients with constipation [41]. Together, these findings are generally consistent with synthesis of new BAs from free cholesterol in the liver to maintain serum BA levels and the hypothesis that increased BA excretion will result in 'mobilisation' of harmful lipids from the liver.

In patients with T2DM in the current study, volixibat $10 \mathrm{mg}$ daily did not alter glucose response to the MTT notably, but there was a nominally significant reduction in fasting blood glucose concentration on days 14 and 28. Increases in insulin and C-peptide levels were slightly less with volixibat than with placebo following the MTT. There was also a trend towards improved insulin sensitivity (increasing HOMA2-\%B and decreasing HOMA2-IR) in the volixibat group. These PD results are promising, given the short duration of treatment and small sample size - limitations that are inherent to phase 1 studies. To the authors' knowledge, no other studies of ASBT inhibition in patients with T2DM have been published, although a study of elobixibat in patients with chronic constipation reported that increases in peak GLP-1 levels, but not morning basal or total AUC levels, on day 12 were greater in the active treatment groups (elobixibat $15 \mathrm{mg}$ and $20 \mathrm{mg}$ once daily) than in the placebo group [39]. Previous studies have shown that diabetes, hypertension, dyslipidaemia and obesity are associated with an increased risk of NASH and of advanced fibrosis among patients with NAFLD $[53,54]$. Thus, lowering serum and hepatic lipid levels and improving the regulation of metabolic function may be of benefit in this patient population.

Treatment with volixibat at daily doses of $0.5-10 \mathrm{mg}$ for 28 days was generally well tolerated. Overall, the safety and PK profiles of volixibat in this study were consistent with those found in the phase 1 study of volixibat in overweight and obese adults, and are broadly consistent with those reported for other ASBT inhibitors $[38-41,52]$. In the present study, there were no deaths, and the one serious TEAE that was reported was considered to be unrelated to the study drug. TEAEs were predominantly gastrointestinal in nature. This was expected given the minimal absorption of volixibat, which results in an increased proportion of BAs reaching the colon. BAs activate intracellular secretory processes in the colon, raising mucosal permeability, increasing mucus secretion and inhibiting chloride hydroxyl exchange [55-57]. In addition, colonic motility is increased as BAs stimulate colonic contractions and reduce colonic transit time $[55,58]$. While this mechanism of action resulted in diarrhoea in $36 / 45$ participants receiving volixibat, compared with $4 / 15$ participants in the placebo group, this TEAE was considered to be mild or moderate in severity and generally decreased over time, with none of the events being reported as serious TEAEs. It should 
also be noted that the composition of serum BA species, including the secondary BAs deoxycholic acid and lithocholic acid, was not altered by volixibat, even though treatment increased total faecal BA excretion by up to eightfold compared with placebo. This finding suggests that treatment does not alter the conditions required for normal biotransformation of primary BAs (predominantly cholic acid and chenodeoxycholic acid) into secondary BAs (deoxycholic acid and lithocholic acid, respectively) by intestinal bacteria.

\section{Conclusions}

In conclusion, daily oral volixibat - a highly potent and selective ASBT inhibitor - was minimally absorbed and generally well tolerated, and increased serum C4 levels and faecal excretion of BAs in HVs and patients with T2DM. In the T2DM cohort, the trends towards increased levels of HDL-C, decreased levels of serum triglycerides and reductions in fasting glucose levels were suggestive of improvements in both lipid and glucose homeostasis. The findings from this study support the initiation of a phase 2 trial (ClinicalTrials.gov Identifier: NCT02787304) to evaluate the efficacy, safety and tolerability of volixibat in adults with NASH.

\section{Additional files}

Additional file 1: Complete inclusion and exclusion criteria. (DOCX $44 \mathrm{~kb}$ )

Additional file 2: Table S1. Glucose metabolism parameters in patients with type 2 diabetes mellitus. (DOCX $67 \mathrm{~kb}$ )

Additional file 3: Table S2. Glucose-insulin metabolism pharmacodynamic parameters in patients with type 2 diabetes mellitus. (DOCX $43 \mathrm{~kb}$ )

\begin{abstract}
Abbreviations
ASBT: Apical sodium-dependent bile acid transporter; AUC: Area under the plasma concentration-time curve; $\mathrm{AUC}_{(0-3)}$ : Area under the effect (serum/ plasma concentration)-time curve from time 0 to $3 \mathrm{~h}$ after the MTT; BA: Bile acids; BMI: Body mass index; C4: 7a-Hydroxy-4-cholesten-3-one;

Cl: Confidence interval; $C_{\text {pre: }}$ Pre-meal tolerance test concentration; EBV: Epstein-Barr virus; $E_{\text {max }}$ : Maximum observed change from the pre-meal tolerance test baseline measurement; FGF: Fibroblast growth factor; FXR: Farnesoid X receptor; GLP: Glucagon-like peptide; $\mathrm{HbA}_{1}$ : Glycosylated haemoglobin; HDL-C: High-density lipoprotein cholesterol; HOMA2\%B: Updated homeostasis model assessment of $\beta$-cell function; HOMA2IR: Updated homeostasis model assessment of insulin resistance; HV: Healthy volunteer; LDL-C: Low-density lipoprotein cholesterol; LS: Least-squares; MTT: Meal tolerance test; NAFLD: Non-alcoholic fatty liver disease; NASH: Non-alcoholic steatohepatitis; PD: Pharmacodynamic; PK: Pharmacokinetic; PRA: Pharmaceutical Research Associates; PYY: Peptide YY; SD: Standard deviation; T2DM: Type 2 diabetes mellitus; TEAE: Treatmentemergent adverse event; US: United States
\end{abstract}

\section{Acknowledgements}

Not applicable.

\section{Funding}

This study was funded by Lumena Pharmaceuticals (part of the Shire group of companies). Initial data analyses were undertaken by Marc Bolt and Minh-Nguyet Duong, who are employees of PRA Health Sciences, which received funding from Lumena Pharmaceuticals. Under the direction of the authors and funded by Shire
International $\mathrm{GmbH}$, writing assistance was provided by Tom Potter of Oxford PharmaGenesis, Oxford, UK. Editorial assistance in formatting, proofreading, copy editing and fact checking was also provided by Oxford PharmaGenesis with funding from Shire International $\mathrm{GmbH}$.

\section{Availability of data and materials}

The data sets generated and/or analysed during the current study are available from the corresponding author on reasonable request. Shire's policy on enhancing data sharing with researchers is available from: http:// www.shiretrials.com/en/our-commitment-to-transparency/data-sharing-withresearchers.

\section{Authors' contributions}

RGT performed the research. BTK and BG contributed to the conduct of the study or collection of data. AAVV and AD contributed to the design of the study. CAK, NL, AD, MP and LA contributed to data analysis and interpretation of data. All authors reviewed the paper and contributed to critical revisions, and approved the final version of the manuscript, including the authorship list.

\section{Ethics approval and consent to participate}

The study was conducted in compliance with the International Conference on Harmonisation E6 Guideline for Good Clinical Practice (Committee for Proprietary Medicinal Products guideline CPMP/ICH/135/95) and was compliant with the European Union Clinical Trial Directive 2001/20/EC. The clinical study protocol, protocol amendments and informed consent forms were reviewed and approved by an independent ethics committee (Stichting Beoordeling Ethiek Biomedisch Onderzoek, Assen, Netherlands). Participants signed the informed consent forms during the pre-study screening visit, within a period of 3 weeks (HVs) or 6 weeks (patients with T2DM) before the first dosing day and before the start of any study-related procedures.

\section{Consent for publication}

Not applicable.

\section{Competing interests}

RGT and AAVV were employees of PRA Health Sciences at the time of this study. CAK, BTK, NL, LA, BG, AD and MP were employees of, or consultants to, Lumena Pharmaceuticals Inc. (part of the Shire group of companies) at the time of this study. MP is currently an employee of Shire and owns stock or stock options. The authors declare that they have no other competing interests.

\section{Publisher's Note}

Springer Nature remains neutral with regard to jurisdictional claims in published maps and institutional affiliations.

\section{Author details}

${ }^{1}$ Early Development Services, Pharmaceutical Research Associates (PRA) Health Sciences, Van Swietenlaan 6, 9728 NZ Groningen, PO Box 8144, 9702 Groningen, KC, Netherlands. ${ }^{2}$ Lumena Pharmaceuticals Inc. (part of the Shire group of companies), 12531 High Bluff Drive, Suite 110, San Diego, CA 92130, USA. ${ }^{3}$ Lexington, USA.

Received: 4 July 2017 Accepted: 22 December 2017

Published online: 05 January 2018

\section{References}

1. LaBrecque DR, Abbas Z, Anania F, Ferenci P, Khan AG, Goh KL, et al. World gastroenterology organisation global guidelines: nonalcoholic fatty liver disease and nonalcoholic steatohepatitis. J Clin Gastroenterol. 2014;48:467-73.

2. Williams CD, Stengel J, Asike MI, Torres DM, Shaw J, Contreras M, et al. Prevalence of nonalcoholic fatty liver disease and nonalcoholic steatohepatitis among a largely middle-aged population utilizing ultrasound and liver biopsy: a prospective study. Gastroenterology. 2011;140:124-31.

3. Musso G, Gambino R, Cassader M, Pagano G. Meta-analysis: natural history of non-alcoholic fatty liver disease (NAFLD) and diagnostic accuracy of noninvasive tests for liver disease severity. Ann Med. 2011;43:617-49.

4. Chalasani N, Younossi Z, Lavine JE, Diehl AM, Brunt EM, Cusi K, et al. The diagnosis and management of non-alcoholic fatty liver disease: practice guideline by the American Association for the Study of Liver Diseases, 
American College of Gastroenterology, and the American Gastroenterological Association. Hepatology. 2012;55:2005-23.

5. Wong WW, Wong GL, Choi PC, Chan AW, Li MK, Chan HY, et al. Disease progression of non-alcoholic fatty liver disease: a prospective study with paired liver biopsies at 3 years. Gut. 2010;59:969-74.

6. Fassio E, Alvarez E, Dominguez N, Landeira G, Longo C. Natural history of nonalcoholic steatohepatitis: a longitudinal study of repeat liver biopsies. Hepatology. 2004;40:820-6.

7. Adams LA, Sanderson S, Lindor KD, Angulo P. The histological course of nonalcoholic fatty liver disease: a longitudinal study of 103 patients with sequential liver biopsies. J Hepatol. 2005;42:132-8.

8. Ekstedt M, Franzen LE, Mathiesen UL, Thorelius L, Holmqvist M, Bodemar G, et al. Long-term follow-up of patients with NAFLD and elevated liver enzymes. Hepatology. 2006:44:865-73.

9. McPherson S, Hardy T, Henderson E, Burt AD, Day CP, Anstee QM. Evidence of NAFLD progression from steatosis to fibrosing-steatohepatitis using paired biopsies: implications for prognosis and clinical management. J Hepatol. 2015;62:1148-55.

10. Matteoni CA, Younossi ZM, Gramlich T, Boparai N, Liu YC, McCullough AJ. Nonalcoholic fatty liver disease: a spectrum of clinical and pathological severity. Gastroenterology. 1999:116:1413-9.

11. Leite NC, Salles GF, Araujo AL, Villela-Nogueira CA, Cardoso CR. Prevalence and associated factors of non-alcoholic fatty liver disease in patients with type-2 diabetes mellitus. Liver Int. 2009;29:113-9.

12. Adibi A, Janghorbani M, Shayganfar S, Amini M. First-degree relatives of patients with type 2 diabetes mellitus and risk of non-alcoholic fatty liver disease. Rev Diabet Stud. 2007:4:236-41.

13. Loomba R, Abraham M, Unalp A, Wilson L, Lavine J, Doo E, et al. Association between diabetes, family history of diabetes, and risk of nonalcoholic steatohepatitis and fibrosis. Hepatology. 2012;56:943-51.

14. Souza MR, Diniz Mde F, Medeiros-Filho JE, Araujo MS. Metabolic syndrome and risk factors for non-alcoholic fatty liver disease. Arq Gastroenterol. 2012;49:89-96.

15. Assy N, Kaita K, Mymin D, Levy C, Rosser B, Minuk G. Fatty infiltration of liver in hyperlipidemic patients. Dig Dis Sci. 2000;45:1929-34.

16. National Cholesterol Education Program Expert Panel. Third report of the National Cholesterol Education Program (NCEP) expert panel on detection, evaluation, and treatment of high blood cholesterol in adults (adult treatment panel III) final report. Circulation. 2002;106:3143-421.

17. Donati G, Stagni B, Piscaglia F, Venturoli N, Morselli-Labate AM, Rasciti L, et al. Increased prevalence of fatty liver in arterial hypertensive patients with normal liver enzymes: role of insulin resistance. Gut. 2004;53:1020-3.

18. Hamaguchi M, Kojima T, Takeda N, Nakagawa T, Taniguchi H, Fujii K, et al. The metabolic syndrome as a predictor of nonalcoholic fatty liver disease. Ann Intern Med. 2005;143:722-8.

19. Marchesini G, Marzocchi R, Agostini F, Bugianesi E. Nonalcoholic fatty liver disease and the metabolic syndrome. Curr Opin Lipidol. 2005;16:421-7.

20. Neuschwander-Tetri BA. Nonalcoholic steatohepatitis and the metabolic syndrome. Am J Med Sci. 2005;330:326-35.

21. Lau E, Carvalho D, Freitas P. Gut microbiota: association with NAFLD and metabolic disturbances. Biomed Res Int. 2015; https:/doi.org/10.1155/2015/979515.

22. European Association for the Study of the Liver, European Association for the Study of Diabetes, European Association for the Study of Obesity. EASLEASD-EASO clinical practice guidelines for the management of nonalcoholic fatty liver disease. J Hepatol. 2016:64:1388-402.

23. Kassirer JP, Angell M. Losing weight-an ill-fated new Year's resolution. N Engl J Med. 1998:338:52-4.

24. Hallsworth K, Avery L, Trenell MI. Targeting lifestyle behavior change in adults with NAFLD during a 20-min consultation: summary of the dietary and exercise literature. Curr Gastroenterol Rep. 2016;18:11.

25. Carvalhana S, Cortez-Pinto H. From obesity to fatty liver/NASH: two parallel epidemics. In: Electronic World Gastroenterology News: the official e-newsletter of the World Gastroenterology Organisation; 2013. http//wwww.worldgastroenterology. org/publications/e-wgn/e-wgn-expert-point-of-view-articles-collection/from-obesityto-fatty-livernash-two-parallel-epidemics. Accessed 20 Jul 2016.

26. Wong RJ, Aguilar M, Cheung R, Perumpail RB, Harrison SA, Younossi ZM, et al. Nonalcoholic steatohepatitis is the second leading etiology of liver disease among adults awaiting liver transplantation in the United States. Gastroenterology. 2015;148:547-55.

27. Banini BA. Nonalcoholic steatohepatitis (NASH) has surpassed hepatitis C as the leading etiology for listing for liver transplant: implications for NASH in children and young adults. Las Vegas: American College of Gastroenterology Annual Scientific Meeting; 2016. (14-19 Oct 2016) Congress abstract 46. https://www.eventscribe.com/2016/ACG/QRcode. asp?Pres=199366. Accessed 20 Dec 2016

28. Schmitz G, Torzewski M, Barlage S, Drobnik W. Atherosclerosis. In: Keri G, Istvan T, editors. Molecular pathomechanisms and new trends in drug research. London; New York: Taylor \& Francis; 2003. p. 413-57.

29. Shneider BL. Intestinal bile acid transport: biology, physiology, and pathophysiology. J Pediatr Gastroenterol Nutr. 2001;32:407-17.

30. Dawson PA, Lan T, Rao A. Bile acid transporters. J Lipid Res. 2009;50:2340-57.

31. Pournaras DJ, Glicksman C, Vincent RP, Kuganolipava S, AlaghbandZadeh J, Mahon D, et al. The role of bile after roux-en-Y gastric bypass in promoting weight loss and improving glycaemic control. Endocrinology. 2012;153:3613-9.

32. Halilbasic E, Claudel T, Trauner M. Bile acid transporters and regulatory nuclear receptors in the liver and beyond. J Hepatol. 2013;58:155-68.

33. Hylemon PB, Zhou H, Pandak WM, Ren S, Gil G, Dent P. Bile acids as regulatory molecules. J Lipid Res. 2009;50:1509-20.

34. Drucker DJ, Nauck MA. The incretin system: glucagon-like peptide-1 receptor agonists and dipeptidyl peptidase-4 inhibitors in type 2 diabetes. Lancet. 2006:368:1696-705.

35. Dunning BE, Foley JE, Ahren B. Alpha cell function in health and disease: influence of glucagon-like peptide-1. Diabetologia. 2005;48:1700-13.

36. Gutzwiller JP, Goke B, Drewe J, Hildebrand P, Ketterer S, Handschin D, et al. Glucagon-like peptide-1: a potent regulator of food intake in humans. Gut. 1999;44:81-6.

37. Cyphert HA, Ge X, Kohan AB, Salati LM, Zhang Y, Hillgartner FB. Activation of the farnesoid $X$ receptor induces hepatic expression and secretion of fibroblast growth factor 21. J Biol Chem. 2012;287:25123-38.

38. Chey WD, Camilleri M, Chang L, Rikner L, Graffner H. A randomized placebocontrolled phase Illb trial of a3309, a bile acid transporter inhibitor, for chronic idiopathic constipation. Am J Gastroenterol. 2011:106:1803-12.

39. Rudling M, Camilleri M, Graffner H, Holst JJ, Rikner L. Specific inhibition of bile acid transport alters plasma lipids and GLP-1. BMC Cardiovasc Disord. 2015;15:75

40. Simren M, Bajor A, Gillberg PG, Rudling M, Abrahamsson H. Randomised clinical trial: the ileal bile acid transporter inhibitor A3309 vs. placebo in patients with chronic idiopathic constipation-a double-blind study. Aliment Pharmacol Ther. 2011;34:41-50.

41. Wong BS, Camilleri M, McKinzie S, Burton D, Graffner H, Zinsmeister AR. Effects of A3309, an ileal bile acid transporter inhibitor, on colonic transit and symptoms in females with functional constipation. Am J Gastroenterol. 2011;106:2154-64

42. Bhat BG, Rapp SR, Beaudry JA, Napawan N, Butteiger DN, Hall KA, et al. Inhibition of ileal bile acid transport and reduced atherosclerosis in apoE-/mice by SC-435. J Lipid Res. 2003;44:1614-21.

43. Vega GL, Grundy SM. Treatment of primary moderate hypercholesterolemia with lovastatin (mevinolin) and colestipol. JAMA. 1987;257:33-8.

44. Kramer W, Glombik H. Bile acid reabsorption inhibitors (BARI): novel hypolipidemic drugs. Curr Med Chem. 2006;13:997-1016.

45. Chen L, Yao X, Young A, McNulty J, Anderson D, Liu Y, et al. Inhibition of apical sodium-dependent bile acid transporter as a novel treatment for diabetes. Am J Physiol Endocrinol Metab. 2012;302:E68-76.

46. Musso G, Gambino R, Cassader M. Cholesterol metabolism and the pathogenesis of non-alcoholic steatohepatitis. Prog Lipid Res. 2013:52:175-91.

47. West KL, Zern TL, Butteiger DN, Keller BT, Fernandez ML. SC-435, an ileal apical sodium co-dependent bile acid transporter (ASBT) inhibitor lowers plasma cholesterol and reduces atherosclerosis in guinea pigs. Atherosclerosis. 2003;171:201-10.

48. Gedulin B, Nikoulina S, Gedulin N, Nazarenkov B, Keller B. Apical sodiumdependent bile acid transport inhibitors (ASBTi) exhibit potent antidiabclic activity in ZDF rats. Diabetologia. 2013;56:S400-1.

49. Rao A, Kosters A, Mells JE, Zhang W, Setchell KDR, Amanso AM, et al. Inhibition of ileal bile acid uptake protects against nonalcoholic fatty liver disease in high-fat diet-fed mice. Sci Transl Med. 2016; https://doi.org/10. 1126/scitransImed.aaf4823.

50. Levy JC, Matthews DR, Hermans MP. Correct homeostasis model assessment (HOMA) evaluation uses the computer program. Diabetes Care. 1998;21:2191-2.

51. Palmer M, Jennings L, Silberg D, Bliss C, Martin P. Volixibat, a minimally absorbed, oral, apical sodium-dependent bile acid transporter (ASBT) 
inhibitor, increases bile acid excretion, reduces serum lipids, is safe and tolerable in overweight and obese subjects, a population characteristic of NASH. Hepatology. 2016;64:574A. (Abstract 1139)

52. Graffner $H$, Gillberg PG, Rikner L, Marschall HU. The ileal bile acid transporter inhibitor A4250 decreases serum bile acids by interrupting the enterohepatic circulation. Aliment Pharmacol Ther. 2016:43:303-10.

53. Neuschwander-Tetri BA, Clark JM, Bass NM, Van Natta ML, Unalp-Arida A, Tonascia J, et al. Clinical, laboratory and histological associations in adults with nonalcoholic fatty liver disease. Hepatology. 2010;52:913-24.

54. Angulo P, Keach JC, Batts KP, Lindor KD. Independent predictors of liver fibrosis in patients with nonalcoholic steatohepatitis. Hepatology. 1999; 30:1356-62

55. Wilcox C, Turner J, Green J. Systematic review: the management of chronic diarrhoea due to bile acid malabsorption. Aliment Pharmacol Ther. 2014;39:923-39.

56. Aldini R, Roda A, Festi D, Sama C, Mazzella G, Bazzoli F, et al. Bile acid malabsorption and bile acid diarrhea in intestinal resection. Dig Dis Sci. 1982;27:495-502.

57. Mekjian HS, Phillips SF, Hofmann AF. Colonic secretion of water and electrolytes induced by bile acids: perfusion studies in man. J Clin Invest. 1971;50:1569-77.

58. Kirwan WO, Smith AN, Mitchell WD, Falconer JD, Eastwood MA. Bile acids and colonic motility in the rabbit and the human. Gut. 1975;16:894-902.

\section{Submit your next manuscript to BioMed Central and we will help you at every step:}

- We accept pre-submission inquiries

- Our selector tool helps you to find the most relevant journal

- We provide round the clock customer support

- Convenient online submission

- Thorough peer review

- Inclusion in PubMed and all major indexing services

- Maximum visibility for your research

Submit your manuscript at www.biomedcentral.com/submit 